ARTICLE

https://doi.org/10.1038/s41467-019-08778-x

\title{
TG-interacting factor 1 (Tgif1)-deficiency attenuates bone remodeling and blunts the anabolic response to parathyroid hormone
}

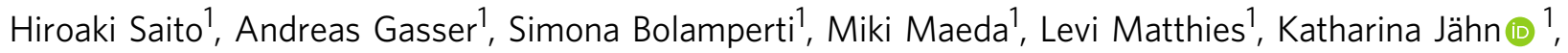
Courtney L. Long ${ }^{1}$, Hartmut Schlüter ${ }^{2}$, Marcel Kwiatkowski², Vaibhav Saini ${ }^{3}$, Paola Divieti Pajevic ${ }^{3,7}$, Teresita Bellido ${ }^{4}$, Andre J. van Wijnen (1) ${ }^{5}$, Khalid S. Mohammad 6 , Theresa A. Guise ${ }^{6}$, Hanna Taipaleenmäki ${ }^{1} \&$ Eric Hesse $e^{1,4}$

Osteoporosis is caused by increased bone resorption and decreased bone formation. Intermittent administration of a fragment of Parathyroid hormone (PTH) activates osteoblastmediated bone formation and is used in patients with severe osteoporosis. However, the mechanisms by which PTH elicits its anabolic effect are not fully elucidated. Here we show that the absence of the homeodomain protein TG-interacting factor 1 (Tgif1) impairs osteoblast differentiation and activity, leading to a reduced bone formation. Deletion of Tgif1 in osteoblasts and osteocytes decreases bone resorption due to an increased secretion of Semaphorin 3E (Sema3E), an osteoclast-inhibiting factor. Tgif1 is a PTH target gene and PTH treatment failed to increase bone formation and bone mass in Tgif1-deficient mice. Thus, our study identifies Tgif1 as a novel regulator of bone remodeling and an essential component of the PTH anabolic action. These insights contribute to a better understanding of bone metabolism and the anabolic function of PTH.

\footnotetext{
${ }^{1}$ Molecular Skeletal Biology Laboratory, Department of Trauma, Hand and Reconstructive Surgery, University Medical Center Hamburg-Eppendorf, Martinistr. 52, D-20246 Hamburg, Germany. ${ }^{2}$ Mass Spectrometric Proteomics Laboratory, Institute for Clinical Chemistry and Laboratory Medicine, University Medical Center Hamburg-Eppendorf, Martinistr. 52, D-20246 Hamburg, Germany. ${ }^{3}$ Endocrine Unit, Massachusetts General Hospital, 55 Fruit St., Boston, MA 02114, USA. ${ }^{4}$ Department of Anatomy and Cell Biology, Indiana University School of Medicine, 635 Barnhill Dr., Indianapolis, IN 46202 , USA. ${ }^{5}$ Department of Orthopedic Surgery, Mayo Clinic, 200 1st St. SW, Rochester, MN 55905, USA. ${ }^{6}$ Division of Endocrinology, Department of Medicine, Indiana School of Medicine, 545 Barnhill Dr., Indianapolis, IN 46202, USA. ${ }^{7}$ Present address: Department of Molecular and Cell Biology, Boston University, School of Dental Medicine, 72 East Concord St., Boston, MA 02118, USA. These authors contributed equally: Hiroaki Saito, Andreas Gasser, Hanna Taipaleenmäki, Eric Hesse. Correspondence and requests for materials should be addressed to E.H. (email: e.hesse@uke.de)
} 


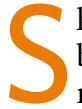
keletal fragility is a growing medical and socioeconomic burden for aging societies ${ }^{1-3}$. Bones are constantly dismantled and rebuilt by matrix-resorbing osteoclasts and bone-forming osteoblasts. This well-balanced process of bone remodeling supports the maintenance of bone quality and stability 2,4 .

Osteoblasts arise from mesenchymal precursor cells and can differentiate into matrix-entrapped osteocytes ${ }^{4}$. Differentiation of osteoblasts is controlled by signaling pathways and regulatory factors, including T-cell factor/lymphoid enhancing factor (Tcf/Lef), Osterix (Osx/Sp7), Runt-related transcription factor 2 (Runx2), zinc finger proteins and homeodomain proteins ${ }^{5-8}$. Osteoblasts and osteocytes provide activation signals to osteoclasts via receptor activator of nuclear factor $-\kappa B$ ligand (RANKL), which binds to the RANK receptor on pre-osteoclasts and mature osteoclasts 9,10 . Osteoblasts can also provide negative signals to osteoclasts by secreting osteoprotegerin (OPG), a soluble RANKL decoy receptor, thereby inhibiting the RANKL-RANK interaction and subsequent osteoclast activation ${ }^{10,11}$. In addition to the RANKL/OPG system, interleukins (ILs), insulin-like growth factors (IGFs) and Ephrin signaling also participate in the osteoblast-osteoclast crosstalk ${ }^{12,13}$.

During aging, bone remodeling often becomes unbalanced with bone resorption exceeding bone formation. This can cause osteoporosis characterized by a decrease in bone mass and bone mineral density (BMD), and fragility fractures ${ }^{3,14}$. Specific osteoporosis treatment includes the reduction of bone resorption using bisphosphonates (e.g., Alendronate, Risedronate or Zoledronate) or an antibody against RANKL (Denosumab) ${ }^{14}$. An alternative approach is to increase bone formation, for instance, by the intermittent administration of a recombinant fragment of human parathyroid hormone containing the first 34 amino acids (hPTH 1-34, Teriparatide, hereafter referred to PTH) ${ }^{2,14,15}$. If administered intermittently in a pharmacological manner, PTH stimulates bone remodeling by increasing the activity of osteoblasts and osteoclasts. This leads to a net increase in bone mass and $\mathrm{BMD}$, thereby reducing the fracture risk ${ }^{15-17}$.

Bone formation is also strongly activated by the canonical Wnt signaling pathway, which was uncovered from rare genetic diseases $^{7}$. For instance, a gain-of-function mutation of the Wnt coreceptor low-density lipoprotein receptor-related protein 5 (LRP5) causes a high bone mass (HBM) phenotype ${ }^{18,19}$. Lrp5 is important for the activation of the canonical Wnt pathway, since binding of Wnt ligands to Lrp5 or 6 and frizzled co-receptors activate the signaling cascade. Once activated, $\beta$-catenin translocates to the nucleus and induces the expression of target genes, including Axin 2 and CyclinD $1^{7}$. Inhibition of Lrp5 signaling can be achieved by the binding of soluble antagonists such as Dickkopf 1 (Dkk1) and Sclerostin ${ }^{20}$. Sclerostin, which is encoded by the SOST gene, is secreted by osteocytes and binds to Lrp4-6 receptors on osteoblasts, thereby antagonizing Wnt signaling ${ }^{21-23}$. Similar to Lrp5, mutations reducing the expression of the SOST gene were identified to cause HBM in sclerosteosis and Van Buchem disease ${ }^{24,25}$. Supported by these findings, a monoclonal anti-sclerostin antibody (Romosozumab) has been developed as a novel bone anabolic therapy, which increases bone mass and strength and decreases the fracture risk ${ }^{26-29}$.

The canonical Wnt pathway and the PTH pathway are not separated and PTH signaling stimulates bone formation by crossactivating the canonical Wnt signaling using various mechanisms, including the decrease of Dkk1 and sclerostin expression ${ }^{30-32}$. Although great advances have been made in the development of novel anti-osteoporosis drugs, it is still very important to further decipher molecular mechanisms underlying the control of physiological and pharmacologically induced bone remodeling to reach a better understanding of these processes. Here, we identify the homeodomain protein TG-interacting factor 1 (Tgif1) as a novel Wnt and PTH target gene and a crucial regulator of osteoblast function. Absence of Tgif1 impairs osteoblast differentiation in vitro and osteoblast activity and bone formation in vivo. Deletion of Tgif1 in osteoblasts and osteocytes also decreases bone resorption due to an increased secretion of the osteoclast-inhibiting factor Semaphorin 3E (Sema3E) ${ }^{33}$. Although the bone anabolic function of Wnt signaling is unaffected by the absence of Tgif1, PTH treatment fails to increase bone mass in mice lacking Tgif1 in the osteoblast lineage. This is at least in part due to an incomplete decrease in the expression of the myocyte enhancer factor 2c (Mef2c) and subsequent sclerostin expression upon PTH treatment. Thus, our study identifies Tgif1 as a novel regulator of bone remodeling and an essential component of the PTH anabolic signaling. These insights contribute to the better understanding of steady state and pharmacologically activated bone remodeling.

\section{Results}

Deletion of Tgif1 impairs bone remodeling. Homeodomain proteins are transcriptional regulators that play important roles in osteoblast differentiation and bone formation ${ }^{5,6}$. To uncover homeodomain proteins with a yet unknown function in the osteoblast lineage, we performed RNA-sequencing in mouse bone marrow stromal cells (BMSCs) and identified Tgif1 as the most abundantly expressed homeodomain protein of unknown function in bone (Fig. 1a). To elucidate the role of Tgif1 in bone, we obtained osteoblasts from mice with a germline deletion of Tgif1 $\left(\text { Tgif } 1^{-/-}\right)^{34}$. Using in vitro differentiation assays, we determined that loss of Tgif1 in osteoblasts decreased the alkaline phosphatase (ALP) activity (Fig. 1b) and the Alp expression (Fig. 1c). Furthermore, Tgifl deficiency impaired the matrix mineralization (Fig. 1d) and reduced the osteocalcin expression (Fig. 1e), demonstrating that Tgif1 promotes osteoblast differentiation.

To rule out the possibility that Tgif2, a paralog of $\operatorname{Tgif} 1^{35}$, is increased in the absence of Tgif1 in a compensatory manner, we quantified the expression of Tgif 2 in Tgif $1^{-/-}$osteoblasts. Tgif2 expression was lower than the expression of Tgif1 (Supplementary Fig. 1a) and unchanged in the absence of Tgif1 (Supplementary Fig. 1a, b), suggesting that the lack of Tgif1 is not compensated by an increased expression of Tgif2.

In order to elucidate the role of Tgifl in bone remodeling, we investigated the bone phenotype of Tgifl-deficient mice. Histomorphometric analysis revealed that the absence of one or both alleles of Tgif1 did not change bone mass (bone volume per tissue volume $(\mathrm{BV} / \mathrm{TV}))$ in the proximal tibiae or the lumbar spine (Fig. 1f, g and Supplementary Fig. 2a, b and Supplementary Tables 1 and 2). However, osteoblast parameters including the number of osteoblasts per bone surface (N.Ob/BS), osteoblast surface per bone surface (Ob.S/BS) and functional indices like the mineral appositions rate (MAR) and the bone formation rate per bone surface (BFR/BS) were significantly decreased in Tgif1 $1^{-/-}$ mice compared to control littermates (Fig. If insets, $1 \mathrm{~g}$ and Supplementary Tables 1 and 2). The greatly impaired osteoblastmediated bone formation was accompanied by a significant reduction of osteoclast parameters, including the number of osteoclasts per bone surface (N.Oc/BS) and the osteoclast surface per bone surface (Oc.S/BS) (Fig. 1g and Supplementary Tables 1 and 2). This led to a reduced eroded surface per bone surface (ES/BS) and therefore to a diminished bone resorption (Fig. 1g and Supplementary Tables 1 and 2). The combined reduction of bone formation and bone resorption caused a gender-independent lowturnover bone phenotype. Thus, these findings confirm the positive role of Tgifl for osteoblast function and bone formation and demonstrate that Tgif1 is a novel regulator of bone remodeling. 
a

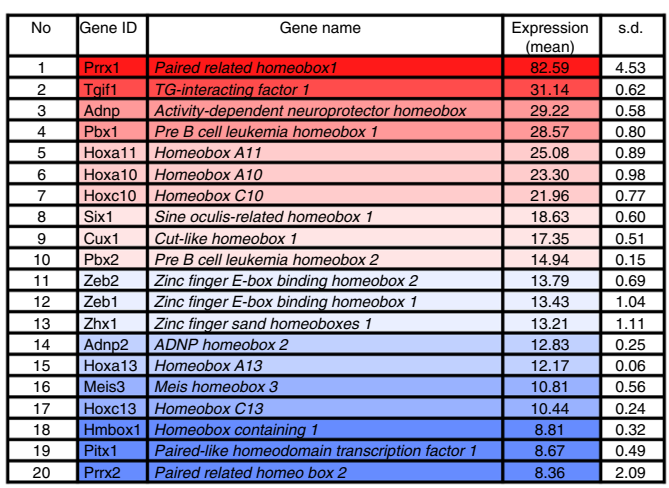

f

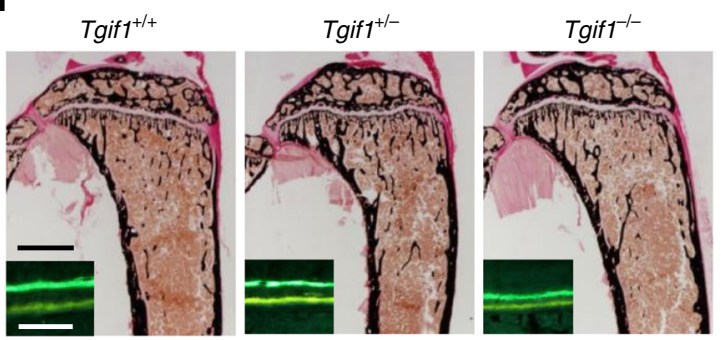

b

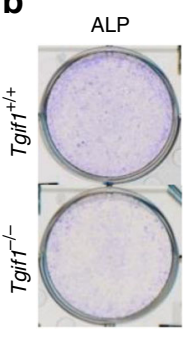

C

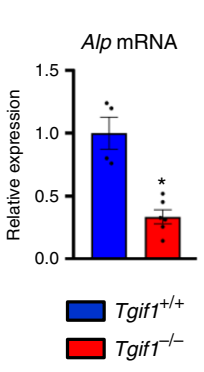

d

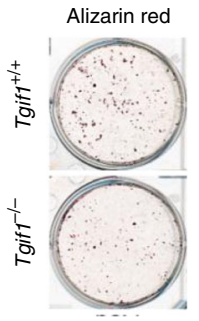

e

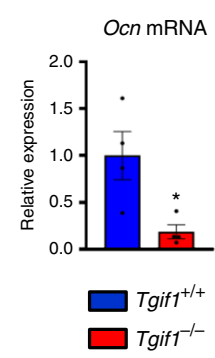

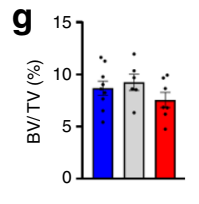
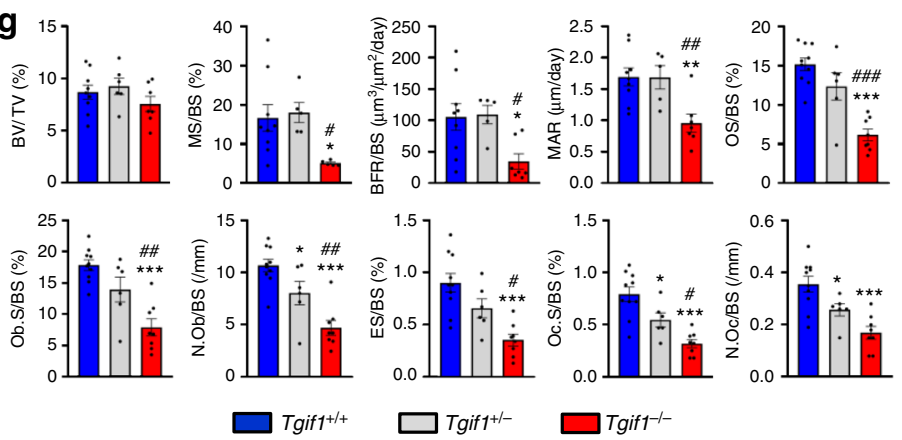

h

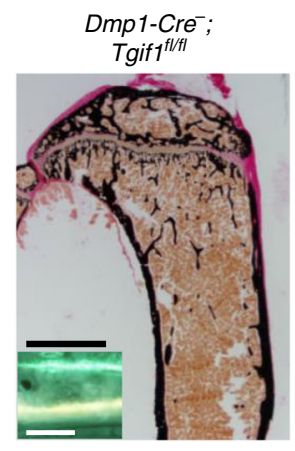

Dmp1-Cre ${ }^{T g}$; Tgiff $^{f / / f t}$

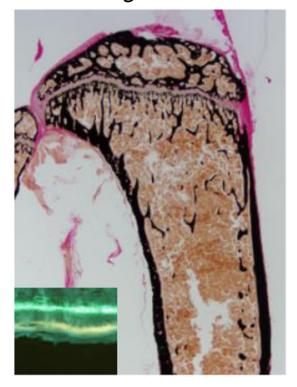

i
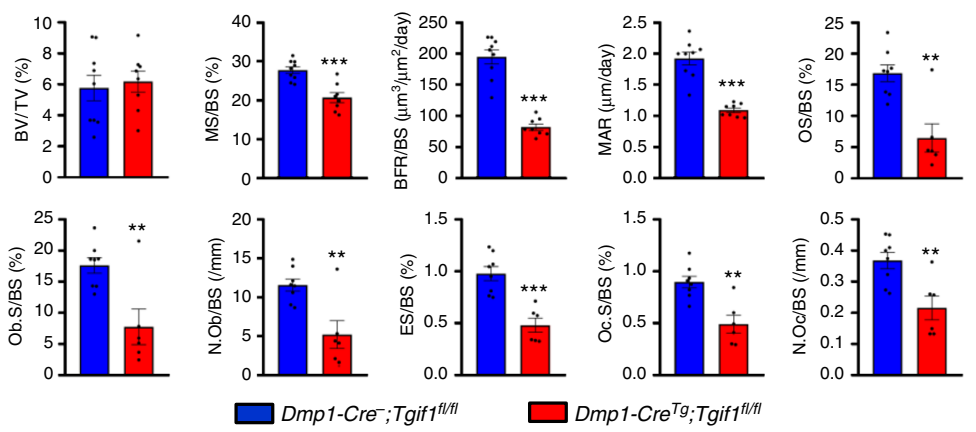

Fig. 1 Deletion of TG-interacting factor 1 (Tgif1) in osteoblasts reduces osteoblast differentiation and bone remodeling. a Homeodomain proteins listed according to their expression in mouse bone marrow stromal cells (BMSCs) during osteoblast differentiation. Expression is indicated as mean values (arbitrary units) with the respective standard deviation (s.d.) $(N=3)$. The first 10 genes are marked in shades of red and the remaining 10 genes are presented in shades of blue. $\mathbf{b}$ Staining of alkaline phosphatase (ALP) activity after in vitro differentiation of Tgif1 ${ }^{+/+}$and Tgif1 $1^{-/-}$calvarial osteoblasts for 14 days (representative image of 3 experiments) and $\mathbf{c}$ relative Alp mRNA expression $(N=4)$. d Determination of matrix mineralization by Alizarin Red staining after 21 days of differentiation (representative image of 3 experiments) and e relative osteocalcin (Ocn) mRNA expression ( $N=4)$. $\mathbf{f}$ Representative images of proximal tibiae of 8-week-old male mice with the genotypes Tgif1 ${ }^{+/+}$, Tgif ${ }^{+/-}$and Tgif1 ${ }^{-/-}$after von Kossa staining and fluorescence double labeling to visualize bone formation (insets). $\mathbf{g}$ Histomorphometric analysis of the proximal tibiae of 8 -week-old male Tgif1 ${ }^{+/+}$, Tgif ${ }^{+/-}$and Tgif1 ${ }^{-/-}$mice $(N=10,6$ and 8). BV/TV bone volume/tissue volume, MS/BS mineralizing surface/bone surface, BFR/BS bone formation rate/bone surface, MAR mineral apposition rate, OS/BS osteoid surface/bone surface, Ob.S/BS osteoblast surface/bone surface, N.Ob/BS number of osteoblasts/bone surface, ES/BS eroded surface/bone surface, Oc.S/BS osteoclast surface/bone surface, N.Oc/BS number of osteoclasts/bone surface. ${ }^{\star} p<0.05$, ${ }^{\star \star} p<0.01,{ }^{\star \star *} p<0.001$ vs.

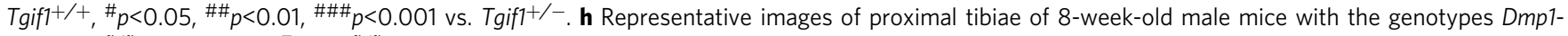
$\mathrm{Cre}^{-} ;$Tgiffl/fl $^{\prime}$ and Dmp1-Cre ${ }^{T g} ; \operatorname{Tgiffl}^{f / f l}$ after von Kossa staining and fluorescence double labeling to visualize bone formation (insets). i Histomorphometric

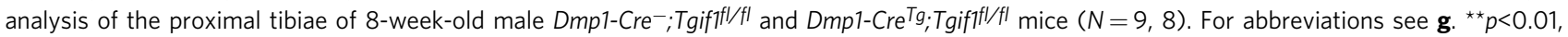

${ }^{\star \star \star} p<0.001$ vs. Dmp1-Cre-; Tgiffl/fll. Scale bars indicate $1 \mathrm{~mm}$ (black) and $50 \mu \mathrm{m}$ (white) f, h. Error bars represent the s.e.m. Two-tailed Student's $t$-test was used to compare two groups $\mathbf{c}, \mathbf{e}, \mathbf{i}$, and analysis of variance (ANOVA) followed by Newman-Keuls post-hoc analysis was used to compare three groups $\mathbf{g}$

To further investigate these findings, we targeted the deletion of Tgif1 to the osteoblast lineage by crossing mice carrying the Tgifi gene flanked by loxP sites (Tgif 1 flffl) 34 $^{34}$ with mice expressing the Cre recombinase downstream of the Osterix (Osx/Sp7) promoter $\left(\mathrm{Osx}-\mathrm{Cre} \mathrm{C}^{T g}\right)^{36}$ or the Dentin matrix protein 1 (Dmp1) regulatory element $\left(\mathrm{Dmpl} 1-\mathrm{Cr} \mathrm{T}^{\mathrm{Tg}}\right)^{37}$. Lack of Tgif1 in osteoblasts

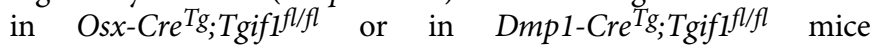

recapitulated the bone phenotype of Tgif1 ${ }^{-/}$mice (Fig. 1h, i and Supplementary Fig. 2c-h and Supplementary Tables 3 and 4), confirming that Tgif 1 in mature osteoblasts and osteocytes is required for the full activation of bone remodeling.

Semaphorin 3E impairs osteoclast differentiation. Interestingly, histomorphometric analysis of bones from mice bearing an 
osteoblast-targeted deletion of Tgif1 revealed a suppression of osteoclast differentiation and bone resorption (Fig. 1i and Supplementary Fig. 2d and Supplementary Table 3 and 4), suggesting a Tgif1-dependent signaling between osteoblasts and osteoclasts. Indeed, in an in vitro co-culture system, Tgif1 $1^{-/-}$osteoblasts were impaired to support osteoclast differentiation demonstrated by a reduced number of tartrate-resistant acid phosphatase (TRAP)positive multinucleated cells (Fig. 2a, b).
Osteoblast-derived factors are well established to contribute to the differentiation and function of osteoclasts ${ }^{12,38,39}$. The most prominent system includes RANKL, which emerges from osteoblasts and binds to its receptor RANK on osteoclasts and can be antagonized by $\mathrm{OPG}^{9,10}$. We therefore quantified the serum concentration of RANKL and OPG in Tgif1 ${ }^{-/-}$and Tgif1 $1^{+/+}$ mice, and calculated the resulting RANKL/OPG ratio but did not detect any differences (Fig. 2c). In addition, the expression of

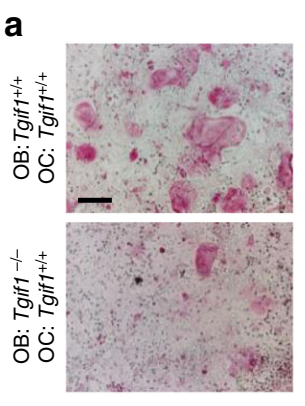

d

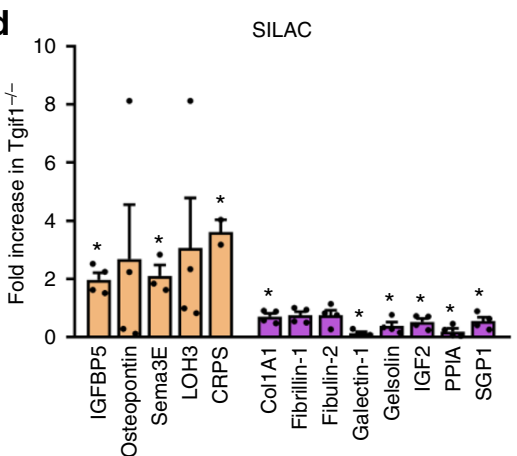

g
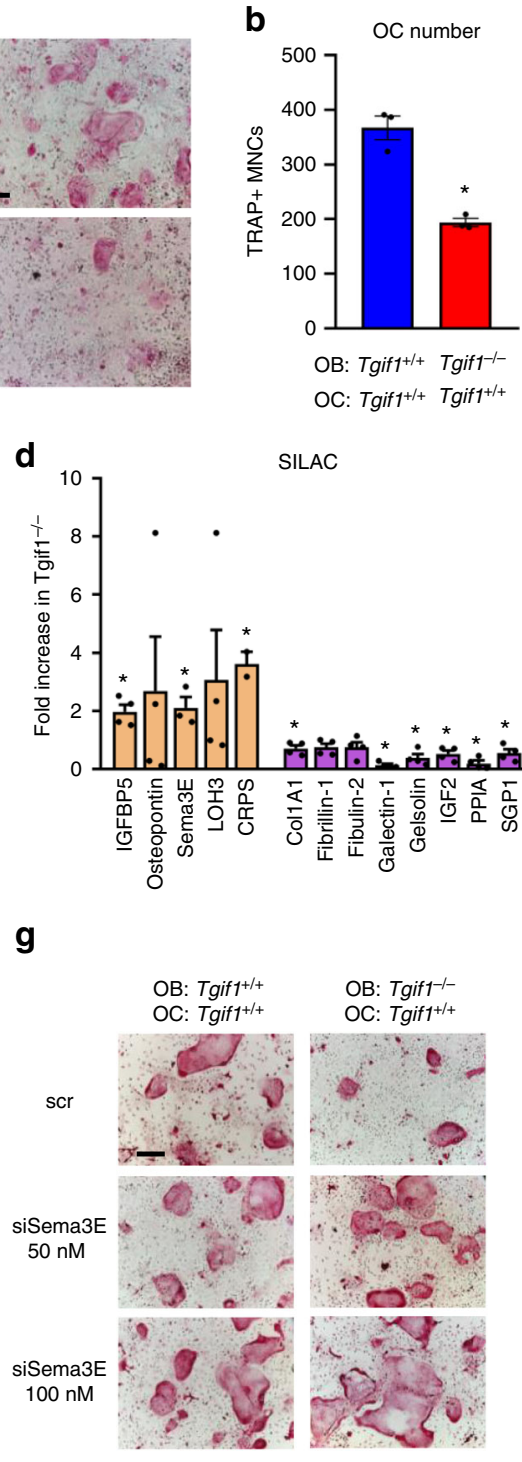
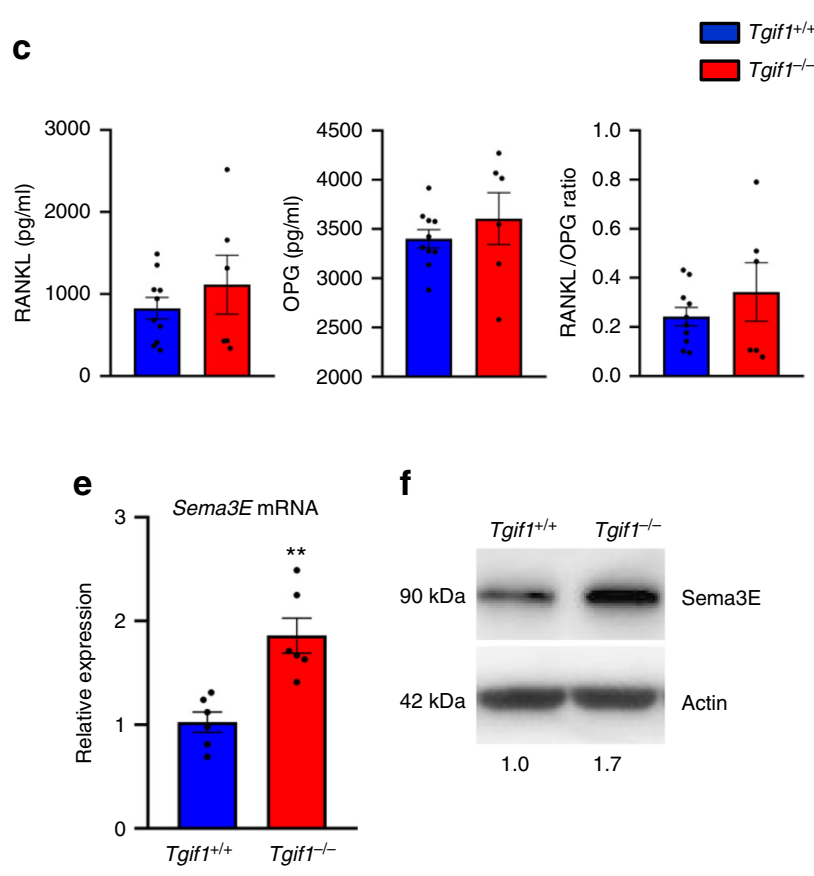

f
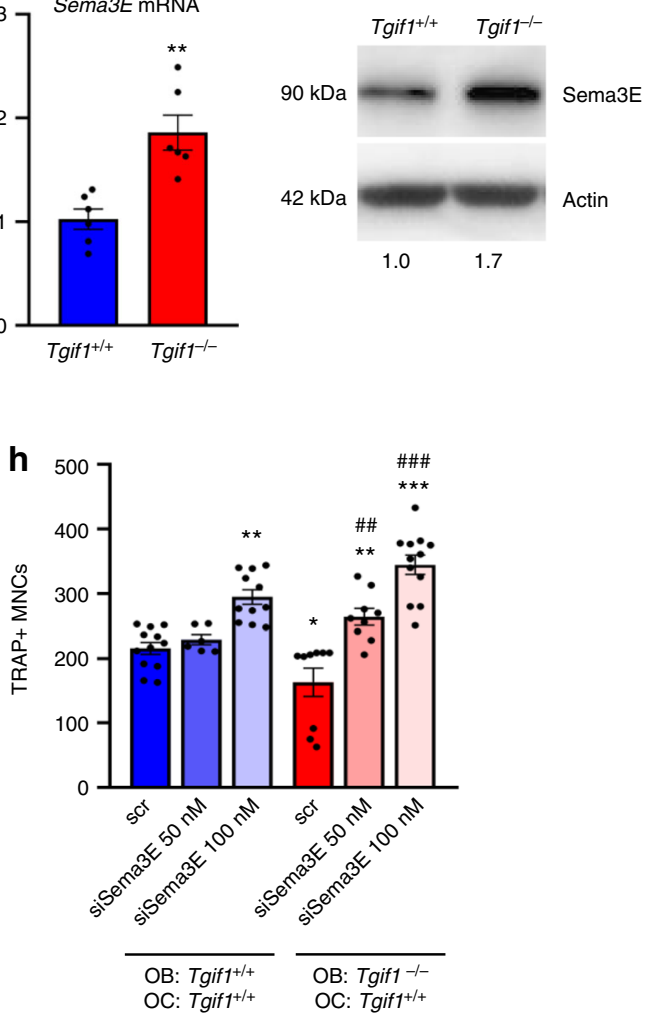

Fig. 2 TG-interacting factor 1 (Tgif1)-deficient osteoblasts inhibit osteoclast differentiation in a Semaphorin 3E (Sema3E)-dependent manner. a Tartrateresistant acid phosphatase (TRAP) staining of osteoclasts (OC) in an osteoblast-osteoclast co-culture with Tgif1 ${ }^{+/+}$osteoclasts and Tgif1 ${ }^{+/+}$or Tgif1 ${ }^{-/-}$ osteoblasts (OB) (representative image of 3 experiments). b Quantification of TRAP-positive multinucleated cells (MNCs) with a minimum of three nuclei $(N=3)$. c Serum concentration of RANKL (receptor activator of nuclear factor NF-kB ligand) and OPG (osteoprotegerin) in Tgif1 ${ }^{+/+}$and Tgif1 ${ }^{-/-}$mice measured by enzyme-linked immunosorbent assay (ELISA) and calculation of the RANKL/OPG ratio $(N=10,6)$. $\mathbf{d}$ Fold increase in proteins secreted by Tgif1 $^{-/-}$calvarial osteoblasts relative to Tgif1 ${ }^{+/+}$calvarial osteoblasts as determined by stable isotope labeling by amino acids in cell culture (SILAC; $N=4)$. e Relative Sema3E mRNA expression in calvarial osteoblasts harvested from Tgif $1^{+/+}$and Tgif $1^{-/-}$mice $(N=6,6) . \mathbf{b}, \mathbf{d}, \mathbf{e}^{*} p<0.05{ }^{* *} p<0.01$ vs. Tgif1 $+/+$ osteoblasts. $\mathbf{f}$ Immunoblot of Sema3E protein expression in calvarial osteoblasts obtained from Tgif1 ${ }^{+/+}$and Tgif $1^{-/-}$mice. Immunoblot for Actin was used as a loading control. Normalized fold expression and molecular weight in kilo Dalton ( $\mathrm{kDa}$ ) are indicated (representative image of 4 experiments). $\mathbf{g}$ Osteoclast differentiation in a co-culture system with Tgif $1^{+/+} \mathrm{OC}$ and Tgif $1^{+/+}$and Tgif1 ${ }^{-/-}$OB transfected with scrambled (scr) control siRNA or Sema3E siRNA (siSema3E) at indicated concentrations (representative image of 4 experiments) and $\mathbf{h}$ quantification of TRAP-positive MNCs ( $N=12$ ).

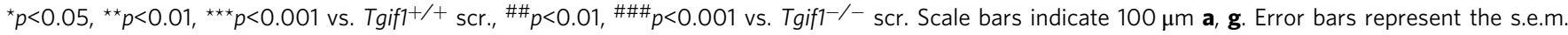
Two-tailed Student's $t$-test was used to compare two groups $\mathbf{b}$-e, and analysis of variance (ANOVA) followed by Newman-Keuls post-hoc analysis was used to compare more than two groups $\mathbf{h}$ 
Rankl and Opg was identical in tibiae, calvarial osteoblasts and in long bone osteoblasts from Tgif1 ${ }^{-/}$mice and control littermates with no change in the Rankl/Opg ratio (Supplementary Fig. 3a-c). Next, we investigated the expression of genes encoding additional factors known to regulate the osteoblast-osteoclast interaction, including Ephrin type-B receptor 2 and 4 (Eph2B, Eph4B), Ephrin $2 B^{40}$, ILs (IL-1, IL-6, IL-11,IL-18) ${ }^{41}$, IGFs (IGF-1,IGF-2) and of their respective binding proteins $(I g f b p-2, \operatorname{Ig} b p-3, \operatorname{Ig} f b p-5)^{13}$ but did not determine an altered expression in Tgif1 ${ }^{-/}$mice (Supplementary Fig. 3d). In order to identify the secreted factor by which Tgif1-/- osteoblasts suppress osteoclast differentiation, we performed an unbiased secretome analysis using stable isotope labeling by amino acids in cell culture (SILAC). Among the factors that were significantly increased in Tgif $1^{-/}$osteoblasts (Fig. 2d), we focused on Sema3E, a class III semaphorin previously shown to inhibit osteoclast formation ${ }^{33}$. Gene expression and immunoblot analysis confirmed a higher expression of Sema3E in Tgif1 $1^{-/-}$ osteoblasts (Fig. 2e, f), suggesting that Tgif1 inhibits Sema3E expression to allow normal osteoclast differentiation. Indeed, small interfering RNA (siRNA)-mediated inhibition of Sema3E expression in Tgif1-/- osteoblasts (Supplementary Fig. 3e) restored the differentiation of co-cultured osteoclasts to the level of control (Fig. 2g, h), demonstrating that Tgifl deficiency increases the expression of the osteoblast-osteoclast signaling molecule Sema3E, leading to an inhibition of osteoclast differentiation.
Tgif1 deficiency in bone does not alter Wnt signaling. Since canonical Wnt signaling is a strong stimulator of bone formation ${ }^{7}$ and because Tgifl has been implicated in oncogenic Wnt signaling, we hypothesized that Tgifl could be an important regulator of this pathway in bone ${ }^{42,43}$. To test this hypothesis, we stimulated osteoblasts with Wnt3a and determined an increase in Tgif1 protein expression (Fig. 3a), thereby establishing Tgif1 as a canonical Wnt target gene. Analysis of the $2.2 \mathrm{~kb}$ upstream region of the Tgif1 promoter revealed the presence of putative Tcf/Lef binding sites, suggesting a transcriptional mechanism underlying the increase in Tgif1 expression upon Wnt stimulation. Indeed, a reporter gene assay revealed an activation of the Tgif1 promoter in osteoblasts upon Wnt3a stimulation (Fig. 3b). Furthermore, reporter gene activation in osteoblasts by Wnt3a (Fig. 3c) or cotransfection with $\beta$-catenin (Fig. $3 \mathrm{~d}$ ) was abolished using truncated promoter fragments lacking Tcf/Lef binding sites. These findings demonstrate that Tgif1 is a Wnt target gene.

Next, we determined if Tgif1 affects the Wnt pathway activity. To address this question, we crossed reporter mice expressing the $\beta$-galactosidase in the presence of activated $\beta$-catenin $(B A T$ $\left.G A L^{T g}\right)$ with $T g i f 1^{-/-}$mice $\left(B A T-G A L^{T g}\right.$;Tgif1-/- $)$. Expression of the $\beta$-galactosidase in $B A T-G A L^{T g}$; $T g i f 1^{-}{ }^{-}$reporter mice as a surrogate for in vivo Wnt activity was unchanged compared to control mice, suggesting that Tgif 1 might not play a functional role in Wnt signaling in bone (Fig. 3e). To ensure the functionality of the system, we harvested BMSCs from long a

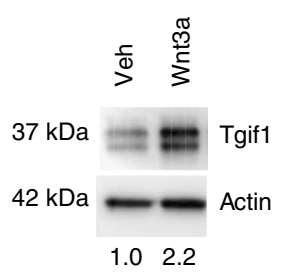

e

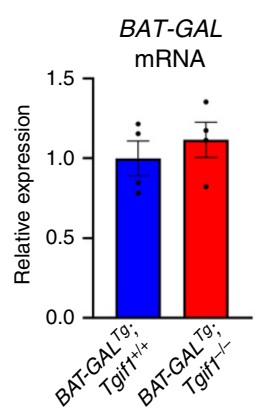

b

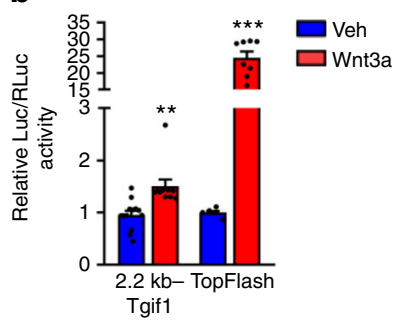

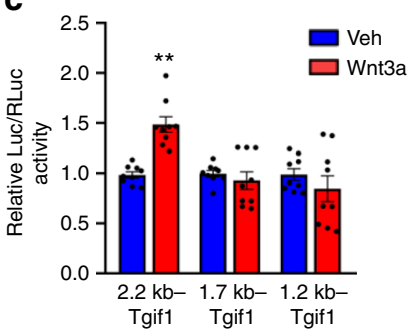

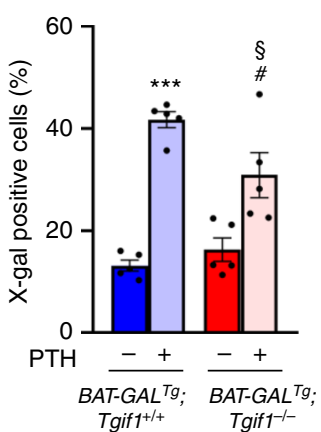

g

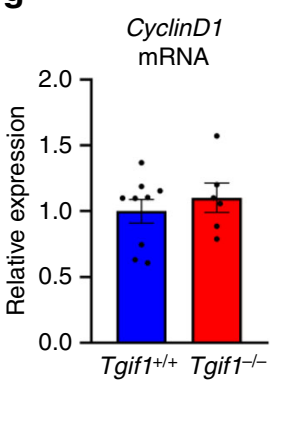

h

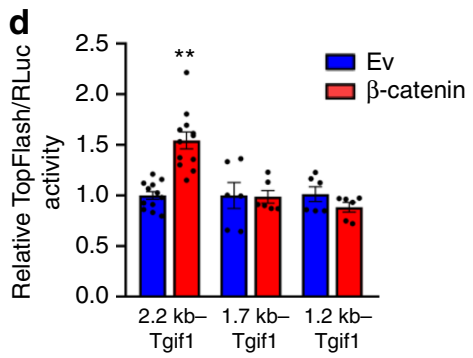

i

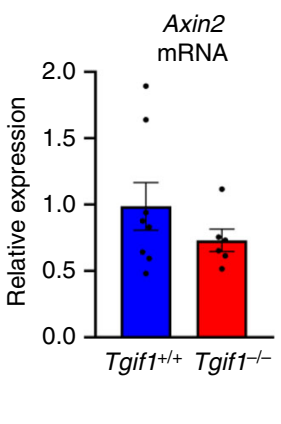

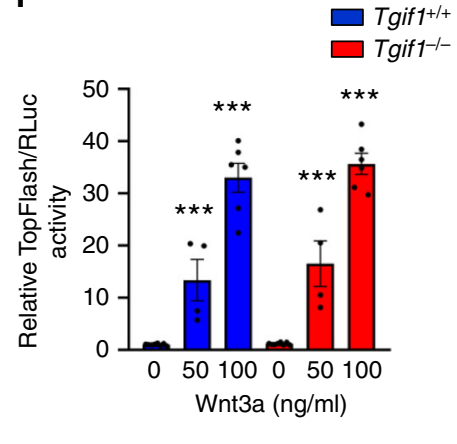

Fig. 3 Deletion of TG-interacting factor 1 (Tgif1) does not alter canonical Wnt signaling. a Immunoblot of Tgif1 protein expression in wild-type calvarial osteoblasts after stimulation with recombinant Wnt3a or vehicle (Veh) for $4 \mathrm{~h}$. Immunoblot for Actin was used as a loading control. Normalized fold expression and molecular weight in kilo Dalton $(\mathrm{kDa})$ are indicated (representative image of 4 experiments). $\mathbf{b}$ Luciferase assays in wild-type calvarial osteoblasts transfected with a $2.2 \mathrm{~kb}$ Tgif1 reporter construct or a Wnt-responsive TopFlash reporter plasmid and stimulated with Veh or Wnt3a for $24 \mathrm{~h}$. c ST2 cells were transfected with a $2.2 \mathrm{~kb}$ Tgif1 reporter construct and different truncations thereof, and stimulated for $4 \mathrm{~h}$ with Veh or Wnt3a. $\mathbf{d}$ ST2 cells were co-transfected with empty vector (Ev) or $\beta$-catenin and the $2.2 \mathrm{~kb}$ Tgif1 reporter construct or truncated forms thereof. b-d ${ }^{\star \star} p<0.01$, ${ }^{\star \star \star} p<0.001$ vs. Veh or Ev control. e Relative $\beta$-galactosidase (BAT-GAL) mRNA expression in tibiae of BAT-GALTg;Tgif1 $+/+$ and BAT-GALTg;Tgif1-/- mice $(N=4,4)$. f Quantification of the fraction of $\beta$-galactosidase-positive bone marrow stromal cells (BMSCs) obtained from BAT-GALTg;Tgif1 ${ }^{\prime /+}$ and BAT-GALTg;Tgif1-/-

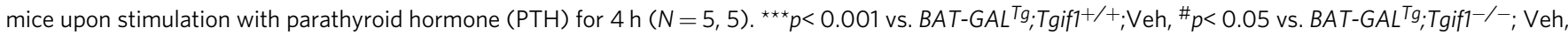
${ }_{5}^{s_{p}<0.05}$ vs. BAT-GAL Tg;Tgif1 ${ }^{+/+} ; \mathrm{PTH}$. g Relative mRNA expression of the Wnt pathway target genes CyclinD1 and $\mathbf{h}$ Axin2 in tibiae of $12-$ week-old male mice of the genotype Tgif1 ${ }^{+/+}$or Tgif1-/- $(N=8,6)$. i Relative activation of the canonical Wnt pathway upon stimulation with increasing concentrations of recombinant Wnt3a in calvarial osteoblasts of the genotypes Tgif1 ${ }^{+/+}$and Tgif1 ${ }^{-/}$- quantified by a TopFlash reporter gene assay $(N=6)$. ${ }^{\star \star \star} p<0.001$ vs. cells of the same genotype without Wnt3a stimulation. Error bars represent the s.e.m. Two-tailed Student's t-test was used to compare two groups $\mathbf{b}$-e, $\mathbf{g}$ $\mathbf{h}$, and analysis of variance (ANOVA) followed by Newman-Keuls post-hoc analysis was used to compare more than two groups $\mathbf{f}, \mathbf{i}$ 
bones of BAT-GAL $L^{T g}$;gif1 $1^{+/+}$and BAT-GAL ${ }^{T g}$;gif1 ${ }^{-/-}$mice and treated the cells with vehicle or $\mathrm{PTH}$, which is known to cross-activate Wnt signaling ${ }^{2}$. Quantification of the $\beta$-galactosidase-positive cells revealed a significant induction of Wnt signaling in BMSCs of both genotypes (Fig. 3f), confirming that the pathway can be activated in this system. The lack of a regulatory role of Tgif1 in Wnt signaling in bone was further confirmed by an unchanged expression of the Wnt target genes CyclinD1 and Axin2 in bones of Tgif1-/- mice compared to control littermates (Fig. 3g, h). Furthermore, Wnt3a activated the TopFlash reporter assay equally in $\mathrm{Tgif1}^{-/-}$and control osteoblasts (Fig. 3i), indicating that in bone the canonical Wnt pathway activity is independent of Tgif1 under steady-state conditions.

Tgifl is dispensable for the Wnt-mediated gain in bone mass. To test the possibility that the absence of Tgif1 affects bone remodeling in response to Wnt activation, we injected Tgif1-/mice and control animals with an antibody against the Wnt pathway inhibitor sclerostin ( $\mathrm{Scl}-\mathrm{Ab}$ ). Quantification of the bone mass revealed that the Scl-Ab-mediated increase in BV/TV was comparable between genotypes (Fig. $4 \mathrm{a}, \mathrm{b}$ and Supplementary Table 5). Next, we crossed two knock-in mouse lines each bearing a HBM mutation of $\operatorname{Lrp} 5$ that are equivalent to mutations found in humans (G170V and $A 213 \mathrm{~V})^{44}$ with Tgif1 ${ }^{-1-}$ animals to constitutively activate Wnt signaling. Consistently, the gain in bone mass in $\operatorname{Lrp} 5^{\mathrm{G} 170 \mathrm{~V} /+}$ and $\operatorname{Lrp} 5^{\mathrm{A} 213 \mathrm{~V} /+}$ mice was independent of Tgif1 (Supplementary Tables 6 and 7). However, a detailed histomorphometric analysis uncovered that activation of the Wnt pathway in response to a Scl-Ab treatment and in mice carrying the $G 170 \mathrm{~V} \mathrm{HBM}$ mutation did increase all osteoblast parameters (OS/BS, Ob.S/BS, N.Ob/BS) and the bone formation rate (BFR/BS) more pronounced in Tgif1 ${ }^{-/}$mice than in control animals (Fig. 4b and Supplementary Tables 5 and 6). Although the exact reason for this observation remains to be elucidated, it could be due to a higher responsiveness on the basis of a low bone turnover remodeling in Tgif1 ${ }^{-/}$mice. However, this effect on osteoblasts did not yield a greater increase in bone mass. Thus, these data confirm that Tgif1 is dispensable to elicit the Wntinduced increase in bone mass.

PTH induces Tgif1 expression through AP1 signaling. Upon binding of PTH to its $\mathrm{G}$ protein-coupled receptor PTHR1, the Gsa-linked cAMP-dependent protein kinase A (PKA) signaling pathway becomes activated ${ }^{45,46}$. Activated PKA induces phosphorylation of the cAMP response element-binding protein (CREB) in the cytoplasm, which translocates to the nucleus and initiates the expression of target genes ${ }^{45,46}$. Members of the activator protein 1 (AP1) family are increased in their expression upon PTH stimulation and participate in regulating osteoblast function and bone formation ${ }^{47}$. Osteoblast activation by PTH not only increases bone formation but also the expression of RANKL,

a

Tgif1 $^{+/+}$

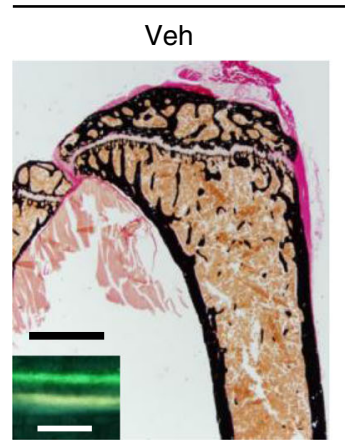

b
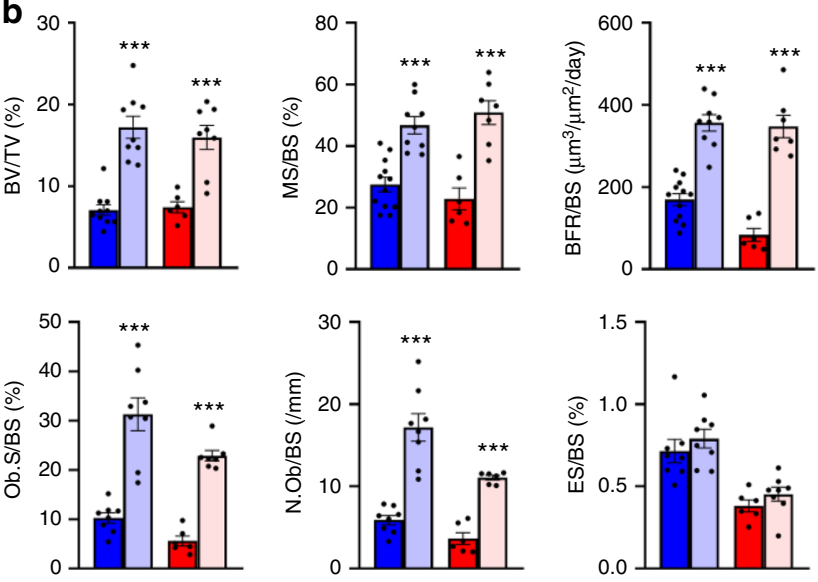

$\mathrm{Scl}-\mathrm{Ab}$

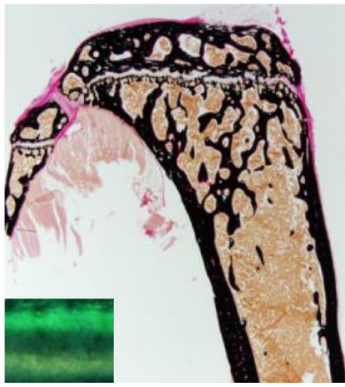

Tgif1 $^{-1-}$

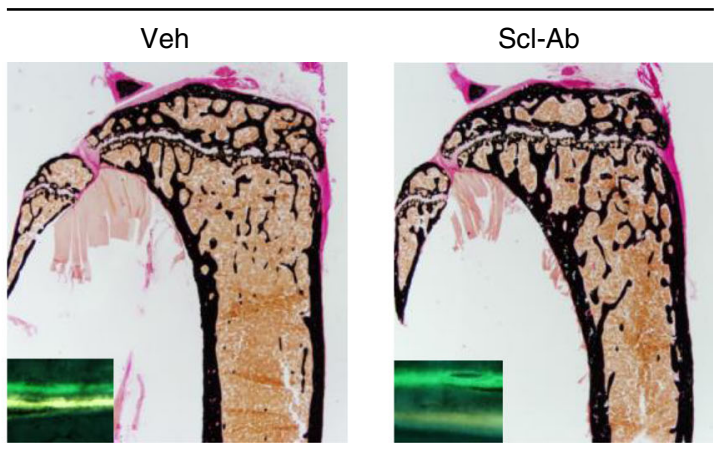

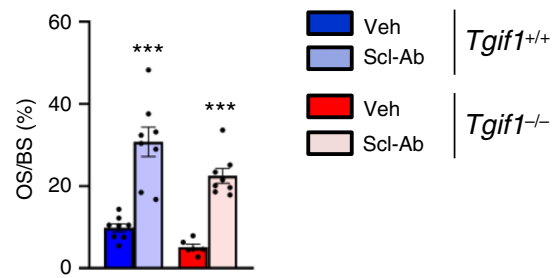
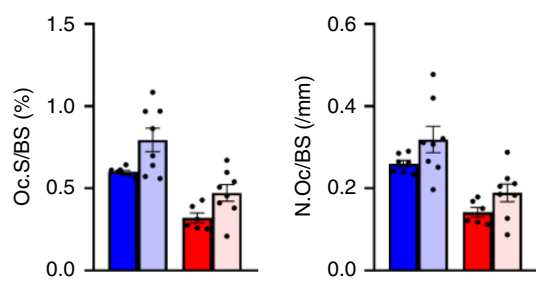

Fig. 4 TG-interacting factor 1 (Tgif1) is dispensable for the bone anabolic function of canonical Wnt signaling. a Representative images of von Kossastained histological sections and fluorescence double labeling to visualize bone formation (insets) of the proximal tibiae of 12 -week-old Tgif $1+/+$ and Tgif1 $^{-/-}$male mice after treatment with anti-sclerostin antibody (Scl-Ab) or vehicle (Veh). Scale bars indicate $1 \mathrm{~mm}$ (black) and $50 \mu \mathrm{m}$ (white).

b Histomorphometric analysis of the proximal tibiae of 12-week-old male Tgif1 ${ }^{+/+}$and Tgif1 ${ }^{-/-}$mice after treatment with $\mathrm{Scl}-\mathrm{Ab}$ or $\mathrm{Veh}\left(\mathrm{Tgif1} 1^{+/+}+\mathrm{Veh}\right.$ : $N=12$, Tgif $^{+/+}+$Scl-Ab: $N=9$, Tgif ${ }^{-/}{ }^{-}+$Veh: $N=6$, Tgif $^{-/}{ }^{-}+\mathrm{Scl}-\mathrm{Ab}: N=8$ ). For abbreviations see the legend to Fig. $1 .{ }^{\star \star} p<0.01,{ }^{\star \star \star} p<0.001$ vs. Veh-treated group of the same genotype. Error bars represent the s.e.m. Statistical analysis was performed using analysis of variance (ANOVA) followed by Newman-Keuls post-hoc test 
which activates osteoclast-mediated bone resorption and causes a high turnover bone remodeling ${ }^{2}$. Since the histomorphometric analysis of Tgif1 ${ }^{-1-}, O s x$-CreTg;Tgif $1^{\text {fllfl }}$ and Dmp1-Cre Tg;Tgif $1^{f l / f l}$ mice revealed a low-turnover bone phenotype (Fig. If-i and Supplementary Fig. $2 c$, d and Supplementary Tables 1-4), we hypothesized that Tgifl might be a component of the PTH signaling cascade. To investigate this hypothesis, we first determined if Tgif1 is a PTH target gene. PTH stimulation increased the Tgif1 protein abundance in calvarial osteoblasts and the Tgif1 mRNA expression in differentiating cells of the osteocyte-derived SW3 cell line (Fig. 5a, b). Furthermore, Tgif1 expression in bones of mice lacking the PTHR1 in mature osteoblasts and osteocytes (Dmp1-CreTg;PTHR $\left.1^{f l f f l}\right)^{48}$ was unchanged under basal conditions, while PTH increased Tgif1 expression in control but not in Dmp1-Cre ${ }^{T g} ; P T H R 1^{f l / f l}$ animals (Fig. 5c). In a reciprocal experiment we determined that the constitutive activation of the PTHR1 in mature osteoblasts and osteocytes (Dmp1caPTHR1) ${ }^{49}$ increased the Tgif1 expression (Fig. 5d) as did the stimulation of calvarial osteoblasts with the PKA activator forskolin (Fig. 5e), demonstrating that Tgif1 expression is induced by the PTH-PKA-pCREB pathway.

To further elucidate the mechanisms by which PTH induces Tgifl expression, we analyzed the $2.2 \mathrm{~kb}$ region of the Tgif1 promoter. This analysis revealed the presence of an AP1 binding site upstream of the Tgifl transcription start site (Fig. 5f), suggesting that PTH might increase Tgif1 expression via AP1 signaling. Indeed, PTH increased the activity of the $2.2 \mathrm{~kb}$ fragment of the Tgif 1 promoter in osteoblasts (Fig. 5g). Progressive truncation of the Tgif1 promoter eliminating the
AP1 binding site (Fig. 5h) and its specific mutation (Fig. 5f, i) abolished the reporter gene activation, demonstrating that PTH induces Tgif1 through PKA-pCREB-AP1 signaling.

PTH increases bone mass in a Tgif1-dependent manner. Since Tgif 1 is a PTH target gene downstream of the PKA-pCREB-AP1 signaling cascade, we proposed that Tgif1 might be involved in the increase in bone mass in response to PTH. To explore this hypothesis, we injected 8-week-old Tgif1-/mice and control littermates with PTH or vehicle intermittently for 4 weeks. In control animals, PTH strongly increased all osteoblast parameters (OS/BS, Ob.S/BS and N.Ob/BS) and bone formation (MS/BS, BFR/BS and MAR) as well as osteoclast parameters (Oc.S/BS and N.Oc/BS) and bone resorption (ES/BS), leading to a high bone mass (BV/TV) phenotype (Supplementary Fig. 4a, b and Supplementary Table 8). In Tgif1 ${ }^{-1-}$ mice, PTH had a mild effect on osteoblast parameters and bone formation (OS/BS, Ob.S/BS, N.Ob/BS, BFR/BS and MAR), while the frequency of mineralizing surfaces per bone surface (MS/BS) remained unchanged. PTH also increased the number of osteoclasts per bone surface (N.Oc/BS) as well as the frequency of eroded surfaces per bone surface (ES/BS) and therefore bone resorption in Tgif1-/- mice. However, these effects were moderate compared to control littermates and, most importantly, did not yield an increase in bone mass (BV/TV) (Supplementary Fig. 4a, b and Supplementary Table 8).

To test whether PTH increases bone mass in mice bearing a Tgif1 deletion in the osteoblast lineage, we injected PTH for a

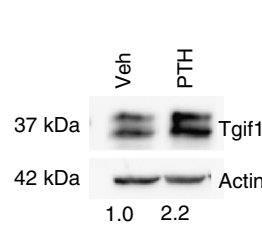

b

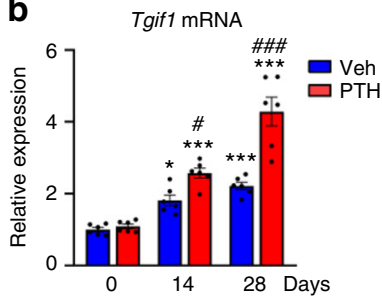

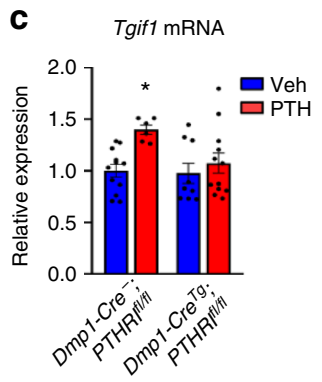

e

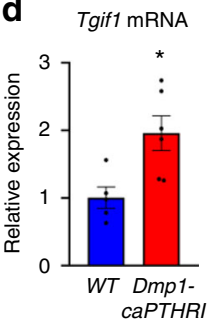

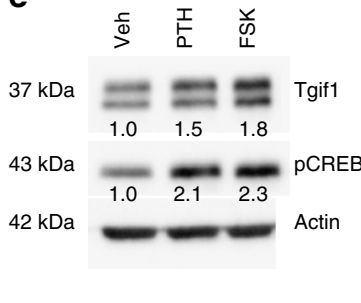

f

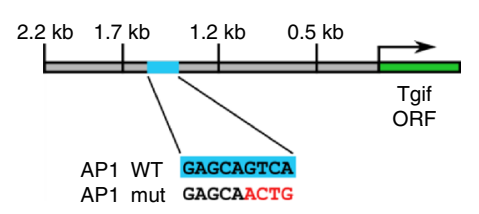

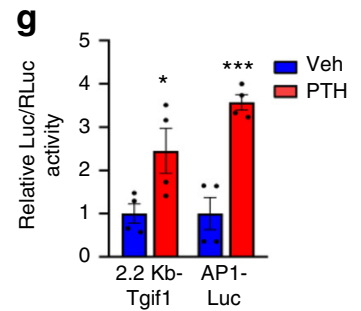
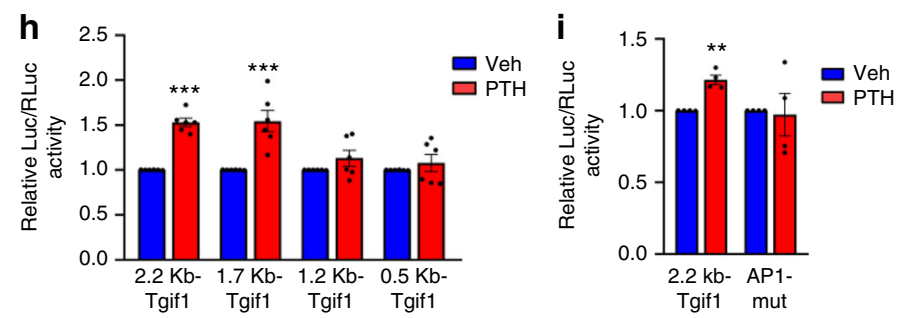

Fig. 5 Parathyroid hormone (PTH) induces TG-interacting factor 1 (Tgif1) expression through activator protein 1 (AP1). a Immunoblot of Tgif1 expression in osteoblasts upon PTH or vehicle (Veh) treatment (representative image of 4 experiments). b Tgif1 expression in differentiating SW3 cells upon PTH or Veh treatment $(N=6,6)$. c Tgif1 expression in tibiae of Dmp1-Cre ${ }^{T g} ; P T H R f^{f / f l}$ mice compared to Dmp1-Cre ${ }^{-} ; \mathrm{PTHR}{ }^{f l / f l}$ control littermates after Veh or PTH injection (Dmp1-Cre-;PTHRfl/fl + Veh, PTH: $N=12,6$, Dmp1-CreTg;PTHRfl/fl + Veh, PTH: N =9, 12). d Tgif1 expression in tibiae of Dmp1-caPTHR1 mice compared to wild-type (WT) littermates $(N=5,6)$. e Immunoblot of Tgif1 and pCREB in osteoblasts after stimulation with PTH, Forskolin (FSK) or Veh (representative image of 3 experiments). $\mathbf{f}$ Schematic of Tgif1 reporter gene constructs. An AP1 binding site (AP1 WT) was mutated (AP1 mut). $\mathbf{g}$ Luciferase activity in osteoblasts transfected with a $2.2 \mathrm{~kb}$ Tgif1 reporter construct or an AP1-Luc reporter plasmid as positive control and stimulated with Veh or PTH (2.2 kb Tgif1+Veh, PTH: $N=4,4$, AP1-Luc+Veh, PTH: $N=4,4)$ h Luciferase activity in ST2 cells transfected with a $2.2 \mathrm{~kb}$ Tgif1 reporter construct or different truncations thereof and stimulated with Veh or PTH (2.2 kb Tgif1+Veh, PTH: N=6, 6, 1.7 kb Tgif1+Veh, PTH: N=6, 6, 1.2 kb Tgif1+Veh, PTH: $N=6,6,0.5$ kb Tgif1+Veh, PTH: $N=6,6)$. i Luciferase activity in ST2 cells transfected with a WT 2.2 kb Tgif1 reporter plasmid or with an inactivated AP1 site (AP1-mut) and stimulated with Veh or PTH (2.2 kb Tgif1+Veh, PTH: N=4, 4, AP1-mut+Veh, PTH: N=4, 4). a, e Immunoblot for Actin was used as a loading control. Normalized fold expression and molecular weight in kilo Dalton (kDa) are indicated. $\mathbf{b}-\mathbf{d}, \mathbf{g}$-i ${ }^{\star} p<0.05,{ }^{\star \star} p<0.01$, ${ }^{\star \star \star} p<0.001$ vs. 0 Days Veh $\mathbf{b}$, Veh $\mathbf{c}, \mathbf{g}$-i or WT d. b $\# p<0.05$, \#\#\#p<0.001 vs. 0 Days PTH. Error bars represent the s.e.m. Two-tailed Student's $t$-test was used to compare two groups $\mathbf{c}, \mathbf{d}, \mathbf{g}-\mathbf{i}$, and analysis of variance (ANOVA) followed by Newman-Keuls post-hoc analysis was used to compare more than two groups $\mathbf{b}$ 
a

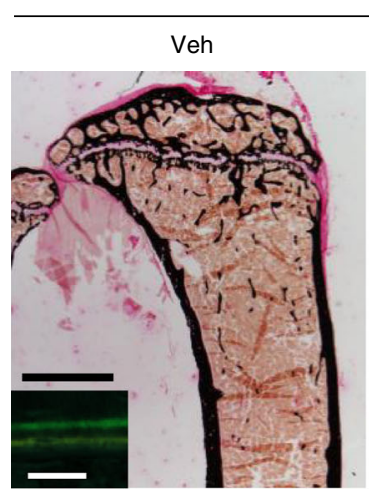

Dmp1-Cre $;$ Tgif1 $^{\text {fl/fl }}$

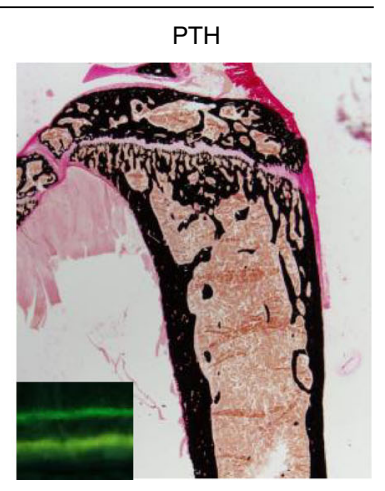

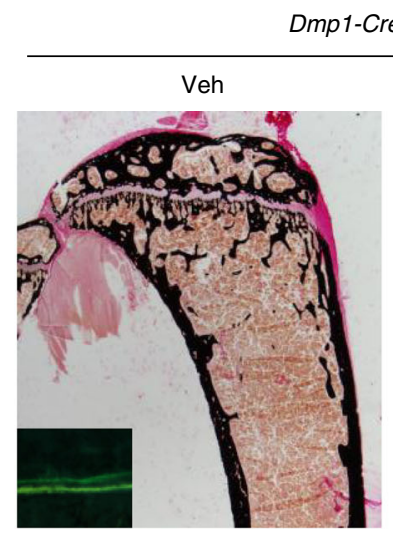

Dmp1-Cre ${ }^{T g} ;$ Tgif1 $^{\text {fllt }}$
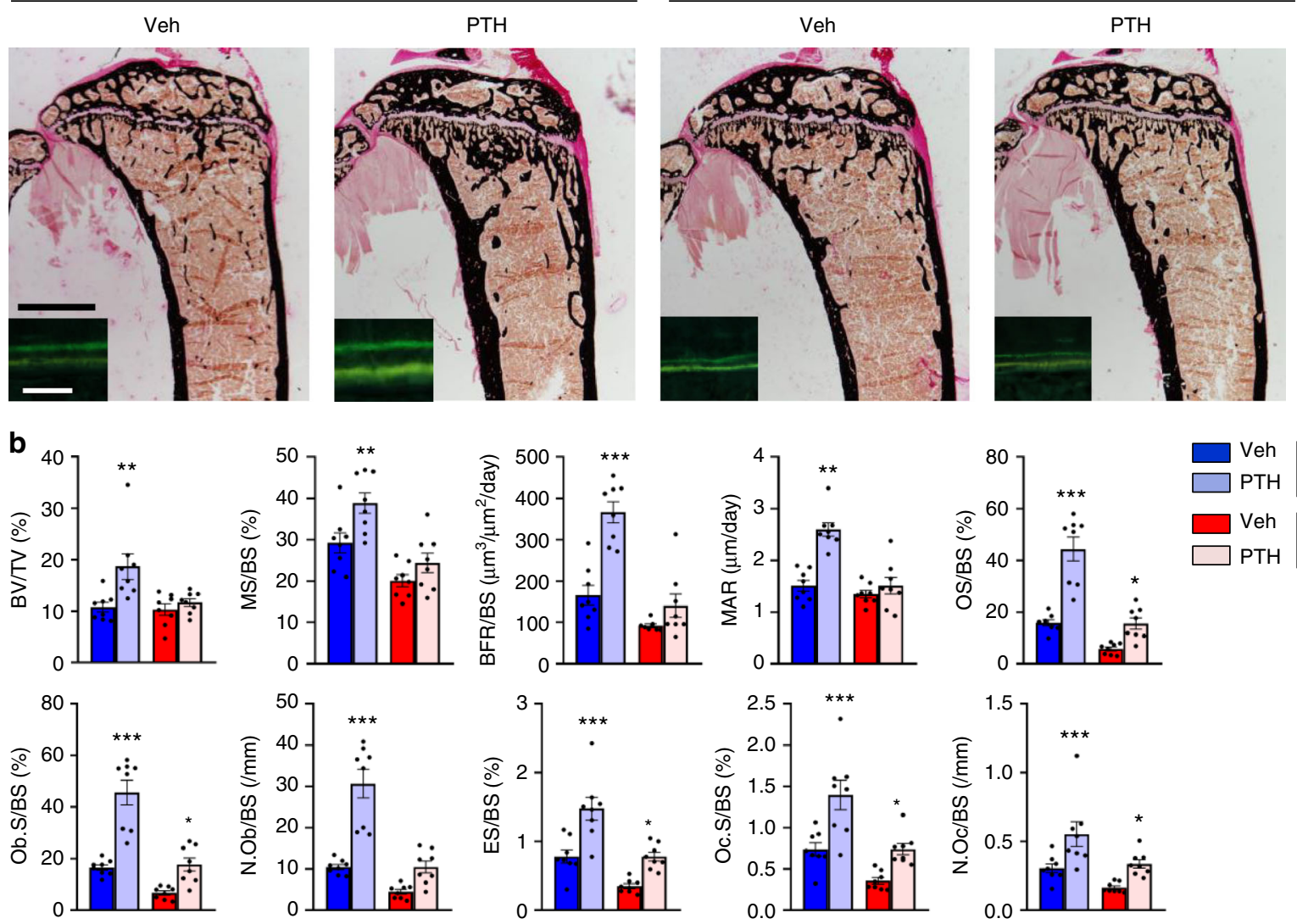

Fig. 6 Parathyroid hormone (PTH) elicits its bone anabolic effect in a TG-interacting factor 1 (Tgif1)-dependent manner. a Representative images of proximal tibiae of 12-week-old male mice with the genotypes Dmp1-Cre-;Tgiffl/ffl and Dmp1-Cre ${ }^{T g} ; T_{g i f f^{f l} / \mathrm{fl}}$ after von Kossa staining and fluorescence double labeling to visualize bone formation (insets). Dmp1-Cre ${ }^{-}$Tgiff ${ }^{f / / f l}$ and Dmp1-Cre ${ }^{T g} ; T_{\text {giff }} f^{f / f l}$ mice were treated with PTH or vehicle (Veh) for 4 weeks. Scale bars indicate $1 \mathrm{~mm}$ (black) and $50 \mu \mathrm{m}$ (white). b Histomorphometric analysis of the proximal tibiae of 12-week-old Dmp1-Cre-; Tgiffl/fl and Dmp1-CreTg;

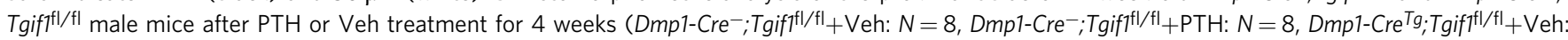
$N=8$, Dmp1-Cre ${ }^{T g} ;$ Tgiff $^{f l / f l}+\mathrm{PTH}: N=8$ ). For abbreviations see the legend to Fig. $1 .{ }^{\star} p<0.05,{ }^{\star \star}{ }^{*}<0.01,{ }^{\star \star \star} p<0.001$ vs. Veh of the same genotype. Error bars represent the s.e.m. Statistical analysis was performed using analysis of variance (ANOVA) followed by Newman-Keuls post-hoc test

4 weeks into 8-week-old Dmp1-Cre ${ }^{T g}$; Tgiff $1^{f l / f l}$ mice and control littermates. Again, in control animals PTH strongly increased all osteoblast (OS/BS, Ob.S/BS and N.Ob/BS) and osteoclast parameters (Oc.S/BS and N.Oc/BS) as well as bone formation (MS/BS, BFR/BS and MAR) and bone resorption (ES/BS) in the context of a highly activated bone remodeling with the consequence of a significant increase in bone mass (BV/TV) (Fig. 6a, b and Supplementary Table 9). In Dmp1-Cre ${ }^{T g}$; $T g i f 1$ fl/fl mice, this effect was attenuated in response to PTH treatment, resulting in a similar phenotype seen in Tgif1 ${ }^{-1-}$ mice. For instance, in contrast to Tgif1 ${ }^{-1-}$ animals the bone formation rate per bone surface (BFR/BS), the mineral apposition rate (MAR) and the number of osteoblasts per bone surface (N.Ob/BS) were not increased in Dmp1-CreTg;Tgifilflfl mice in response to PTH. Consistently, bone mass (BV/TV) was also not changed in Dmp1$\mathrm{Cre}^{T g} ;$ Tgif $^{\text {flf } f l}$ mice (Fig. 6a, b and Supplementary Table 9). These findings support the notion that Tgif1 is a critical component of the PTH-mediated increase in bone formation and bone mass accrual.

PTH reduces sclerostin expression in part through Tgifl. The bone anabolic effect of PTH is in part mediated through crossactivation of other pathways including canonical Wnt signaling $31,32,46,50,51$. For instance, stimulation of osteoblasts and osteocytes with PTH decreases the expression of the canonical
Wnt inhibitors Dkk1 and sclerostin, the product of the SOST gene ${ }^{30,31,52,53}$. Since the Wnt pathway is a strong inducer of bone formation ${ }^{15}$, antagonizing inhibitors of this pathway leads to an increase in bone mass. To investigate whether Tgif1 is implicated in the PTH-mediated cross-activation of the Wnt pathway, we quantified the expression of Dkk1 and sclerostin in the long bones of Tgif1 ${ }^{-1-}$ mice and control littermates $4 \mathrm{~h}$ after injection of PTH or vehicle. Gene expression analysis revealed that PTH fully reduced the expression of $D k k 1$ in bones of $T g i f 1^{-1-}$ mice and control littermates (Fig. 7a), while the expression of Sost mRNA was only partially and not statistically significantly reduced in mice lacking Tgif1 (Fig. 7b). To confirm this observation, we performed immunohistochemistry to detect the abundance of sclerostin in the bones of mice treated with PTH. Consistent with the results of the gene expression analysis, sclerostin protein expression in osteocytes was fully abrogated by PTH in control animals and only partially reduced in Tgif $1^{-/}$ mice (Fig. $7 \mathrm{c}$ ). These findings demonstrate that Tgif1 in mature osteoblasts and osteocytes is necessary for the full PTH-mediated inhibition of sclerostin expression.

To unravel the molecular mechanism by which Tgif 1 contributes to the suppression of sclerostin expression, we analyzed the $8 \mathrm{~kb}$ upstream region of the Sost promoter and identified 11 putative sites to which Tgif1 could bind (Supplementary Fig. 5a). Tgifl forms complexes with other factors and 
a

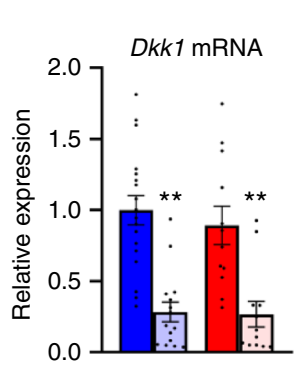

d

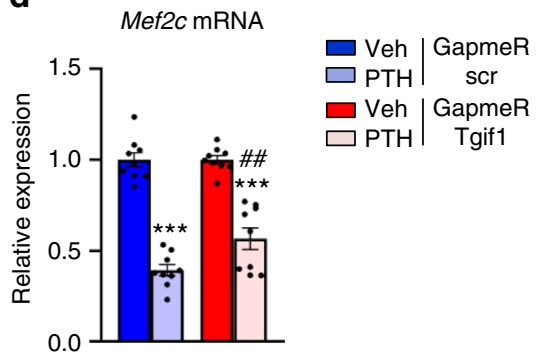

b

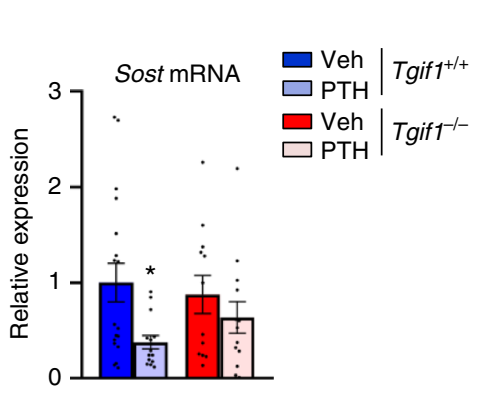

e

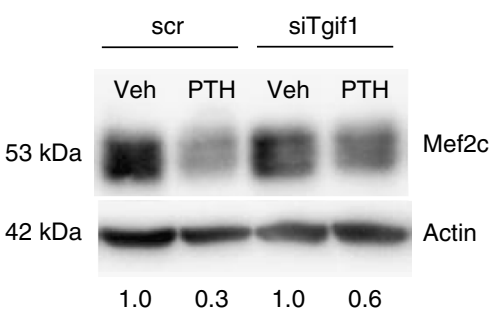

C

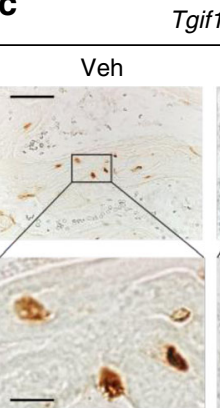

gif1 $1^{+/+}$

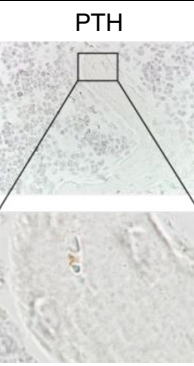

$\mathbf{f}$

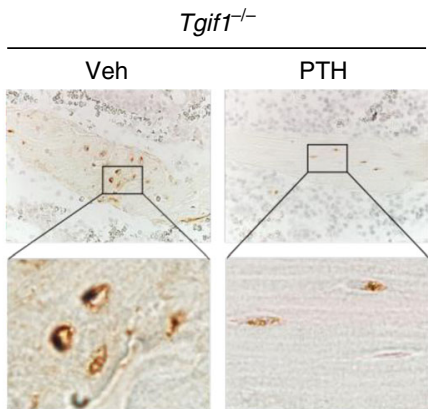

g

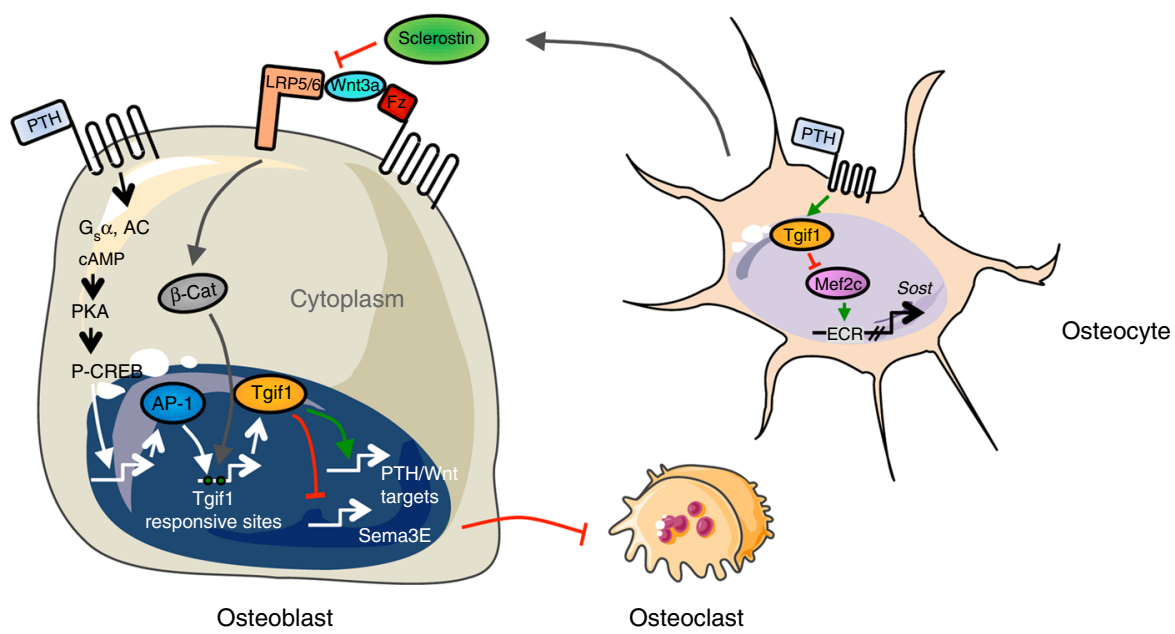

Fig. 7 Suppression of sclerostin expression by parathyroid hormone (PTH) depends in part on TG-interacting factor 1 (Tgif1). a Relative expression of Dkk1 mRNA and of $\mathbf{b}$ Sost mRNA in tibiae of Tgif1 ${ }^{+/+}$and Tgif1 ${ }^{-/-}$mice $4 \mathrm{~h}$ after injection of PTH or vehicle (Veh) $\left(\mathrm{Tgif1}^{+/+}+\mathrm{Veh}: \mathrm{N}=18, \mathrm{Tgif1}{ }^{+/+}+\mathrm{PTH}\right.$ : $N=14$, Tgif1 ${ }^{-/-}+$Veh: $N=12$, Tgif1 ${ }^{-/-}+$PTH: $\left.N=13\right)$. c Immunohistochemical staining of sclerostin expression in tibiae of Tgif1 ${ }^{+/+}$and Tgif1 ${ }^{-/-}$mice after treatment with PTH or Veh. Scale bars indicate $50 \mu \mathrm{m}$ (lower magnification, upper panel) and $10 \mu \mathrm{m}$ (higher magnification, lower panel). d Relative expression of Mef2c mRNA in UMR-106 cells. Cells were transfected with scrambled (scr) control GapmeR or GapmeR targeting Tgif1. After 48 h, cells were stimulated with PTH or Veh for $8 \mathrm{~h}(\mathrm{~N}=3)$. e Immunoblot demonstrating the protein abundance of Mef2c in UMR-106 cells. Cells were transfected with scr siRNA or siRNA targeting Tgif1 (siTgif1). After $48 \mathrm{~h}$, cells were stimulated with PTH or Veh for $8 \mathrm{~h}$. Immunoblot for Actin was used as a loading control. Normalized fold expression and molecular weight in kilo Dalton ( $\mathrm{kDa}$ ) are indicated (representative image of 3 experiments). $\mathbf{f}$ UMR-106 cells were transfected with scr GapmeR or GapmeR targeting Tgif1 and $24 \mathrm{~h}$ later with a hSOST-ECR5 reporter construct. After $8 \mathrm{~h}$, cells were stimulated with PTH or Veh for $16 \mathrm{~h}$. Shown is the relative luciferase activity (GapmeR scr+Veh: $N=3$, GapmeR scr+PTH: N=3, GapmeR Tgif1+Veh: N=3, GapmeR Tgif1+PTH: $N=3) . \mathbf{a}, \mathbf{b}{ }^{\star} p<0.05,{ }^{\star \star} p<0.01$ vs. Veh of the same genotype. $\mathbf{d}, \mathbf{f}{ }^{\star \star \star} p<0.001$ vs. the respective GapmeR+Veh, ${ }^{\#} p<0.005$ vs. GapmeR scr $+\mathrm{PTH}$. Error bars represent the s.e.m. Statistical analysis was performed using analysis of variance (ANOVA) followed by Newman-Keuls post-hoc test. $\mathbf{g}$ Schematic drawing of the function of Tgif1 in osteoblasts, osteocytes and in response to the activation of the canonical Wnt and PTH bone anabolic pathways

represses gene transcription ${ }^{54}$. We therefore hypothesized that PTH might increase the Sost promoter occupancy by Tgif1 and attenuate its activity. To test this hypothesis, we performed chromatin immunoprecipitation (ChIP) of Tgif1 in the OCY454 osteocyte-like cell line $2 \mathrm{~h}$ after incubation with PTH or vehicle. The ChIP assays revealed that Tgifl associates with all sites investigated, except binding sites 8 and 10 (Supplementary Fig. 5b). Binding to site 9 was detectable but did not reach significance (Supplementary Fig. 5b). In contrast to our hypothesis, treatment with PTH caused a dissociation of Tgif1 from all sites with the exception of sites 7 and 9 where no significance was reached (Supplementary Fig. 5b). As a positive control, binding of Tgif1 to the promoter of retinoic acid receptor alpha (Rar alpha) and its dissociation upon PTH treatment was confirmed (Supplementary Fig. 5b) ${ }^{55}$. These observations suggest that Tgif1 might not have a suppressive effect on the Sost promoter activity in response to PTH treatment and that other mechanisms might exist. 
In osteocytes, Mef2 transcription factors activate a distant enhancer of the SOST gene corresponding to the region that is deleted in Van Buchem disease ${ }^{32}$. Furthermore, PTH-mediated inhibition of sclerostin expression in response to $\mathrm{PTH}$ stimulation is largely due to a decrease in Mef2c expression ${ }^{32}$. Interestingly, while the distal enhancer of the SOST gene does not contain Tgif1 consensus sequences, the Mef2c promoter contains several Tgifl binding sites. This indicates that Tgifl might contribute to the control of sclerostin expression in an indirect manner by participating in the repression of Mef2c transcription in response to PTH treatment. Indeed, PTH suppressed Mef2c mRNA expression and protein abundance to a much lesser extent in Tgif1-deficient cells (Supplementary Fig. 6a, b) compared to controls (Fig. 7d, e). To determine the functional relevance of this observation, we analyzed the activity of a gene reporter construct containing the Mef2-responsive distal enhancer element of the human SOST gene ${ }^{32}$. Consistent with our findings, PTH strongly suppressed the reporter gene activity in control cells but to a significantly lower degree in cells in which the expression of Tgif1 had been restricted (Fig. $7 \mathrm{f}$ ), demonstrating the functional role of Tgif1 in regulating the SOST gene activity.

In summary, in response to PTH treatment, Tgif1 dissociates from the SOST promoter and inhibits the expression of Mef2c. Due to a reduced abundance of Mef2c, a distant enhancer element of the SOST gene containing Mef2c binding sites becomes less activated, causing a decrease in sclerostin expression and an increase in bone formation. In the absence of Tgif1, this regulatory cascade is disturbed, leading to an impaired decrease of sclerostin expression in response to PTH treatment and a lower bone formation. Thus, Tgif1 is an important mediator of the gain in bone mass in response to PTH treatment.

\section{Discussion}

In this study, we identified Tgif1 as a novel stimulator of osteoblast differentiation and function. Bone remodeling depends on Tgif1, since absence of Tgif1 in osteoblasts causes low bone turnover due to a decreased osteoblast function and bone formation. The impaired bone formation is accompanied by an osteoblast-mediated decrease in osteoclast activity and bone resorption. Since the amount of bone resorbed equals the amount of bone formed, bone mass is unchanged in Tgifl-deficient mice.

Interestingly, osteoclast differentiation and function is reduced upon deletion of Tgif1 in osteoblasts. After changes in the RANKL/OPG system, in Ephrin signaling and in the expression of interleukins and insulin-like growth factors were excluded, Sema3E was found to be highly expressed by Tgif1 $1^{-/}$osteoblasts and to mediate the suppression of osteoclast differentiation. Although an osteoblast-targeted deletion of Sema3E is needed to determine whether this alleviates the reduced bone resorption in mice lacking Tgif1 in osteoblasts, our findings underscore the important function of Sema3E in bone cell interaction.

In breast cancer, Tgif1 expression correlated with a poor prognosis and supported the Wnt1-driven cancer development ${ }^{42}$. In colorectal cancer, Tgif1 is indispensable for canonical Wnt activation and promotes the disease through Wnt activation ${ }^{43}$. The role of Tgif1 in oncology let us to speculate whether Tgif1 might also be implicated in Wnt signaling in bone. Consistent with the findings in cancer cells, Wnt signaling increased Tgif1 expression in osteoblasts. This increase was mediated by $\beta$-catenin-Tcf/Lef signaling since eliminating Tcf/Lef binding sites in the Tgif1 promoter abolished the transcriptional activation. In contrast to cancer cells, absence of Tgif1 in bone or osteoblastic cells did not change the basal activity of the Wnt pathway in vitro or in vivo. We further determined that Tgifl is dispensable for Wnt-mediated increase in bone mass. This discrepancy to cancer might be due to differences in the molecular regulation of $\mathrm{Wnt}$ signaling pathways under malignant versus benign conditions and suggests that Wnt signaling might function differently in bone than in other organs.

PTH binds to the PTH/PTHrP receptor (PTHR1), a class II G protein-coupled receptor that activates several pathways, including the Gq/11-linked phosphatidyl inositol-specific phospholipase $\mathrm{C}$-protein kinase $\mathrm{C}$ signaling pathway and the Gsa-linked cAMPdependent protein kinase A (PKA) signaling pathway ${ }^{45,46}$. Our in vitro and in vivo data demonstrate that Tgif1 expression in osteoblasts and osteocytes is downstream of the PTHR1. Further analysis revealed that Tgif1 expression is induced by PKA-CREB signaling. Downstream of PTHR1, PKA signaling leads to the phosphorylation of CREB, which induces the expression of AP1 transcription factors ${ }^{45-47}$. AP1 signaling is a mediator of the anabolic effect of $\mathrm{PTH}^{47}$. The functional relevance of an AP1 binding site in the Tgif1 promoter was demonstrated using truncations and a specific mutation in reporter assays. These experiments establish Tgif1 as a PTH target gene downstream of the PTHR1-PKA-pCREB-AP1 cascade.

Similar to the Wnt pathway, Tgifl expression is increased by PTH treatment. Although Tgif1 is dispensable for the bone anabolic function of Wnt signaling, we addressed the question of whether Tgif1 might be implicated in the increase in bone mass by PTH treatment. This hypothesis was supported by a lowturnover bone remodeling in the absence of Tgif1, which represents a somehow opposite phenotype obtained by intermittent administration of PTH. In a pharmacological context, PTH augments osteoblast function and bone formation but also the production of RANKL, thereby activating osteoclasts and bone resorption $^{2}$. This causes a high turnover bone remodeling with a net gain in bone mass. Indeed, daily injections of PTH into mice with a germline deletion of Tgif1 or a targeted deletion of Tgif1 in osteoblasts and osteocytes prevented the increase in bone mass compared to control littermates. However, absence of Tgif1 did not fully abrogate all effects of PTH on bone remodeling and some differences were noticed between the two mouse models of Tgif1 deletion. These findings indicate that Tgif1 contributes to many but not all effects downstream of PTHR1 signaling. Most importantly, in the absence of Tgif1 in the osteoblast lineage, the residual effects of $\mathrm{PTH}$ on bone are insufficient for bone mass accrual.

PTH exerts its bone-forming function also in part through cross-activation of canonical Wnt signaling 46 . For instance, PTH inactivates glycogen synthase kinase- $3 \beta$ (GSK3 $\beta)^{50}$ and stabilizes $\beta$-catenin ${ }^{51}$. PTHR1 forms a complex with Lrp6 upon binding of $\mathrm{PTH}$, thereby disintegrating the destruction complex ${ }^{51}$. In this context, Lrp6 in osteoblasts was shown to be essential for the anabolic effect of $\mathrm{PTH}^{51}$. In osteocytes, $\mathrm{PTH}$ reduces the expression of the Wnt inhibitors $D k k 1^{30}$ and sclerostin ${ }^{49}$. Inhibition of sclerostin expression in osteocytes is mediated by Mef2 transcription factors that control the activity of a distant enhancer element of the SOST gene ${ }^{32}$. In detail, PTH signaling leads to a PKA-mediated phosphorylation and inhibition of the saltinducible kinase 2 (SIK2). This reduces the phosphorylation of histone deacetylases 4 and 5 (HDAC4/5), which translocate to the nucleus and inhibit the Mef2c-driven sclerostin expression ${ }^{56}$. Evidence for the physiological role of sclerostin inhibition as an important component of the PTH anabolic effect is provided by in vivo studies, demonstrating that PTH decreases sclerostin expression in wild-type mice. Furthermore, the PTH-induced gain in bone mass was blunted in mice overexpressing or lacking sclerostin, demonstrating that sclerostin is an important component of anabolic PTH signaling 57 . However, other studies have shown a normal or even enhanced response to PTH treatment in SOST knockout mice ${ }^{46}$. Consistent with these findings, the 
increase in bone mass by PTH in mice overexpressing human sclerostin under the control of the $8 \mathrm{~kb}$ fragment of the Dmp1 promoter was comparable to control littermates ${ }^{58}$. These controversies might be explainable by the use of transgenes obtained from different species, the use of various artificial promoter elements to drive the overexpression or other technical aspects. Nevertheless, although the exact contribution of the downregulation of sclerostin expression in response to $\mathrm{PTH}$ is not yet fully elucidated, this mechanism is likely to be an important component of the PTH bone anabolic effect.

In order to determine whether the PTH-mediated crosstalk with the Wnt pathway could be impaired in Tgif1 $1^{-1-}$ mice in which PTH treatment did not increase bone mass, we investigated the Dkk1 and sclerostin expression in bones from Tgif1-1- mice and control littermates. The basal expression of $D k k 1$ and Sost was comparable between Tgif1 $1^{-/-}$and control mice. Of note, PTH treatment suppressed the Dkk1 expression in animals of both genotypes strongly and equally but the Sost expression was only partially decreased in Tgif1 $1^{-/}$mice compared to control littermates. This observation was confirmed in bone sections at the protein level using immunohistochemistry. Although the incomplete downregulation of sclerostin expression by PTH in $T g i f 1^{-/}$mice was moderate, it might at least in part contribute to the blunted bone anabolic effect of PTH in Tgif1deficient mice.

Although Tgif1 physically associates with the Sost promoter, an inhibitory effect on sclerostin expression is less likely due to the displacement of Tgif1 from its consensus binding sites in response to $\mathrm{PTH}$ stimulation. However, our findings demonstrate that Tgifl contributes to the PTH-mediated repression of Mef2c expression. By this mechanism, Tgifl has an indirect inhibitory effect on sclerostin expression. In the absence of Tgif1, PTH stimulation reduces Mef2c expression incompletely, allowing the remaining Mef2c to bind to a distant enhancer element of the Sost gene, leading to a residual transcriptional activity and sclerostin expression.

In summary, the PTH-PKA-pCREB-AP1 pathway and canonical Wnt signaling increase Tgifl expression in osteoblasts and osteocytes (Fig. 7g). Tgif1 supports osteoblast differentiation, function and bone formation. By suppressing the expression of Sema3E, Tgif1 facilitates bone resorption and therefore physiological bone remodeling. In osteocytes, Tgif1 contributes to the inhibition of Mef2c expression and thereby to the decrease of sclerostin expression in response to $\mathrm{PTH}$ treatment. Thus, our study identified Tgif1 as a novel regulator of bone remodeling and an essential component of the PTH anabolic action.

\section{Methods}

Mouse models. Mice with a germline deletion of Tgif1 and a loxP-flanked Tgif1 gene have been reported previously ${ }^{34}$. To delete Tgif1 in osteoblasts in vivo, mice expressing the Cre recombinase under the control of the Osterix (Osx/SP7) promoter $\left(\mathrm{Os} x-\mathrm{Cre} e^{T g}\right)^{36}$ or an $8 \mathrm{~kb}$ fragment of the murine Dentin matrix protein 1 (Dmp1) (Dmp1-Cre $\left.e^{T g}\right)^{37}$ promoter were crossed with mice in which exons 2 and 3 of the Tgifl gene are flanked by loxP sites $\left(\text { Tgifl }^{f l /+}\right)^{34}$. The resulting mice of the genotype Osx-Cre ${ }^{T g}$; Tgif $1^{f l /+}$ were mated with Tgif1 ${ }^{f l /+}$ mice to obtain Osx-Cre ${ }^{T g}$; Tgif1 $1^{f / f l}$ mice with a conditional deletion of Tgif1 in osteoblasts. Osx-Cre $e^{T}$; Tgif $^{+/ /+}$ mice were used as control to exclude a non-specific phenotype caused by the Cre expression. Since no bone phenotype was observed in Dmp1-Cre ${ }^{+}$mice, Dmp1-CreTgifl ${ }^{f l f l}$ mice were used as controls. Mice carrying the Lrp $5^{\mathrm{G} 170 \mathrm{~V} /+}$ and $\operatorname{Lrp} 5^{\mathrm{A} 213 \mathrm{~V} / \mathrm{+}}$ HBM mutations were obtained from Jackson Laboratories (Bar Harbor). Mice with a conditional ablation of the PTHR1 controlled by a $10 \mathrm{~kb}$ fragment of the Dmp1 promoter (Dmp1-Cre $e^{T g}$; $P$ THR $\left.1^{f l / f l}\right)$ and mice expressing a constitutively active PTHR1 (caPTHR1) regulated by an $8 \mathrm{~kb}$ fragment of the Dmp1 promoter (Dmp1caPTHR1) have been described previously ${ }^{48,49}$. For bone anabolic studies, a recombinant fragment of human parathyroid hormone (PTH $1-34 ; 100 \mu \mathrm{g} / \mathrm{kg}$ of body weight, Biochem) was administered intraperitoneally 5 times a week for 4 weeks. Anti-sclerostin antibody (Scl-Ab; kindly provided by Novartis and Mereo BioPharma) was delivered intravenously at a concentration of $100 \mu \mathrm{g} / \mathrm{kg}$ once a

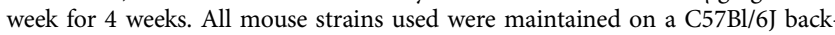
ground. Sample sizes were determined according to the standards used in the field.
All experimental animals that were alive by the time of analysis were included in the study. Mice of the same genotype were randomized to the treatment or control group. Investigators were not blinded to the group allocation during the experiment, but assessment of the outcome by bone histomorphometry was performed in a blinded manner. The study received approval by the local authority for animal welfare and experiments were conducted in compliance with all relevant ethical regulations for animal testing and research.

Bone analyses. Mice were injected 7 and 2 days before sacrifice with calcein (20 $\mathrm{mg} / \mathrm{kg}$ ) and demeclocycline $(20 \mathrm{mg} / \mathrm{kg}$; both Sigma-Aldrich), respectively. Tibiae and the fourth lumbar vertebral bodies (L4) were collected and fixed in 3.7\% phosphate-buffered saline (PBS)-buffered formaldehyde. For histomorphometric analysis, tibiae and L4 were embedded in methylmethacrylate. Toluidine blue, von Kossa and TRAP staining were performed using $4 \mu \mathrm{m}$ sagittal sections. Quantitative bone histomorphometric measurements were performed according to standard protocols $^{59}$ using an OsteoMeasure system (OsteoMetrics). Microcomputed tomography $(\mu \mathrm{CT})$ was used for bone analyses. Distal and midshaft femora were analyzed using high-resolution $\mu \mathrm{CT}$ with a fixed isotropic voxel size of $15.6 \mu \mathrm{m}$ (70 $\mathrm{kV}$ at $114 \mu \mathrm{A}, 400 \mathrm{~ms}$ integration time; Viva80 micro-CT; Scanco Medical AG). All analyses were performed on digitally extracted bone tissue using three-dimensional distance techniques ${ }^{60}$.

\section{Cell culture and ex vivo osteoblast differentiation assays. BMSCs were} obtained from three different mice. For each cell preparation, cells were plated at a density of 10,000 cells $/ \mathrm{cm}^{2}$ in 3 parallel cultures and cultured for 2 days in $\alpha$ minimum essential medium ( $\alpha$-MEM; Life Technologies) containing $10 \%$ fetal bovine serum (FBS; Life Technologies) and $100 \mathrm{U} / \mathrm{ml}$ penicillin; $100 \mu \mathrm{g} / \mathrm{ml}$ streptomycin (P/S; Life Technologies). Prior to RNA isolation, BMSCs from 3 parallel cultures were pooled. The mouse stromal cell line ST2 was purchased from DSMZ (Cat. No.: ACC 333) and maintained in RPMI-1640 medium (Life Technologies) supplemented with $10 \%$ FBS and P/S. The rat osteosarcoma cell line UMR-106 was purchased from ATCC (Cat. No.: CRL-1661) and maintained in Dulbecco's modified Eagle's medium (DMEM; Life Technologies) with $10 \%$ FBS and P/S. The osteocyte-derived OCY454 cell line has been reported previously ${ }^{61}$ and the osteocyte-derived IDG-SW3 cell line was kindly provided by Dr. Lynda Bonewald. Cells were maintained and expanded in culture dishes coated with rat tail type I collagen (Corning) at $33^{\circ} \mathrm{C}$ in complete medium (a-MEM, $10 \% \mathrm{FBS}$, and P/S) in the presence of $10 \mathrm{U} / \mathrm{ml}$ interferon-gamma. Differentiation was performed at $37^{\circ} \mathrm{C}$ in the absence of interferon-gamma but with the addition of $0.2 \mathrm{mM} \mathrm{L}$-ascorbic acid (Sigma) and $5 \mathrm{mM} \beta$-glycerophosphate (Millipore). For calvarial osteoblast cultures, calvariae were dissected from 1- to 3-day-old mice and digested sequentially in a-MEM containing $0.1 \%$ collagenase and $0.2 \%$ dispase (both Roche). Cell fractions 2 to 4 were combined and expanded in $\alpha$-MEM containing $10 \% \mathrm{FBS}$ and $\mathrm{P} / \mathrm{S}$. Osteoblast differentiation was induced by supplementing $\alpha-$ MEM with $50 \mu \mathrm{g} / \mathrm{ml} \mathrm{L}$-ascorbic acid and $5 \mathrm{mM} \beta$-glycerophosphate. Osteoblast differentiation was determined by alkaline phosphatase (ALP) and Alizarin Red staining after fixing the cells in $4 \%$ neutrally buffered formaldehyde solution. For ALP staining, cells were incubated with naphthol ASMX/Fast Blue (both from Sigma-Aldrich) in Tris- $\mathrm{HCl}$ solution at $\mathrm{pH} 8.4$ for $15 \mathrm{~min}$ at room temperature. To detect matrix mineralization, Alizarin Red staining was performed with $40 \mathrm{mM}$ Alizarin Red S (AR-S; Sigma-Aldrich) solution at $\mathrm{pH} 4.2$ for $10 \mathrm{~min}$ at room temperature.

Long bone osteoblasts were isolated from femora and tibiae of 8-10-week-old mice. After removing muscles in sterile PBS, bone marrow was flushed and bones were cut in small pieces. Bone pieces were digested with $0.1 \%$ collagenase for $2 \mathrm{~h}$ at $37^{\circ} \mathrm{C}$ and plated in a-MEM containing $10 \% \mathrm{FBS}$ and P/S. Outgrowing osteoblasts were detached with trypsin after 1 week and cultured until confluence (2-3 weeks) Bone marrow macrophages (BMMs) were isolated from the bone marrow of 8 week-old C57Bl/6J mice. Non-adherent cells were collected after $3 \mathrm{~h}$ of incubation on plastic and cultured in $\alpha$-MEM containing $10 \%$ FBS, P/S and macrophage colony stimulating factor $(100 \mathrm{ng} / \mathrm{ml}$, PeproTech). For osteoblast-osteoclast cocultures, long bone osteoblasts were plated on 96-well plates and stimulated with Vitamin D and Prostaglandin E2 (PGE2). One day later, BMMs were plated over osteoblasts. Cultures were terminated after 5 days, fixed and stained with TRAP for $10 \mathrm{~min}$ at $37^{\circ} \mathrm{C}$. All reagents of the TRAP solution (Naphthol-ASMX-Phosphate, Fast Red Violet LB-Salt and $N, N$-Dimethylformamid) were purchased from Sigma. No cell line used is listed in the database of commonly misidentified cell lines maintained by ICLAC. Cell lines used in this study have not been authenticated but have been regularly tested for mycoplasma contamination using the PCR Mycoplasma Test Kit (PromoKine).

Transfection of siRNAs and GapmeRs. Scrambled siRNAs and siRNAs targeting mouse Sema3E and rat Tgif1 were purchased from Dharmacon. Scrambled GapmeRs and GapmeRs targeting rat Tgifl were purchased from Qiagen. Long bone osteoblasts were transfected using the NEON Transfection system (Thermo Fisher Scientific). UMR-106 rat osteosarcoma cells were transfected using Lipofectamine 3000 (Life Technologies) 
DNA constructs and Luciferase assays. To mutate the Tgif1 promoter reporter construct (kindly provided by S.J. Brandt), the AP1 binding site was modified using the QuickChange II XL site-directed mutagenesis kit (Agilent). ST2 cells were transfected with progressively truncated Tgifl reporter constructs and a reporter construct bearing a mutated AP1 binding site along with a Renilla reporter using Lipofectamine reagent (Life Technologies) according to the supplier's recommendations. TopFlash $\beta$-catenin luciferase reporter (Addgene) containing 7 concatenated Tcf/Lef binding sites were co-transfected with a plasmid encoding $\beta$-catenin as well as a Renilla luciferase reporter plasmid in calvarial osteoblasts using the NEON transfection system. UMR-106 cells were transfected with the hSOST-ECR5-luc reporter construct ${ }^{32}$ and a Renilla luciferase reporter plasmid using Lipofectamine 3000. Luciferase assays were performed using the Dual-Glo Luciferase Reporter Gene Assay System (Promega) according to the instructions provided by the manufacturer. Firefly luciferase activity was normalized to Renilla luciferase activity.

Gene expression analyses. Total RNA was isolated from mouse bones using TRIzol reagent (Life Technologies) and from cultured cells using the RNeasy Plus Mini kit (Qiagen) according to the manufacturer's instructions. Complementary DNA (cDNA) was synthesized from $1 \mu \mathrm{g}$ of total RNA using the ProtoScript First Strand cDNA Synthesis Kit (NEBioLabs). Quantitative real-time PCR (qRT-PCR) was performed using SYBR Green (Bio-Rad). After normalization to TATAbinding protein $(\mathrm{Tbp}) \mathrm{mRNA}$, relative expression levels and fold induction of each target gene were calculated using the comparative CT $(\Delta \Delta \mathrm{CT})$ method. All oligonucleotides used for qPCR analysis are listed in Supplementary Table 10.

RNA sequencing. Next-generation RNA sequencing was performed using three distinct mouse bone marrow-derived mesenchymal stromal cell populations. Each cell preparation was plated in three cultures and combined prior to analysis. Oligo dT purified mRNA was used to generate cDNAs that were indexed using the standard TruSeq Kits 12-Set A and 12-Set B on Illumina RTA version 1.17.21.3. Read mapping was performed using a robust pipeline involving MAPRSeq v.1.2.1, TopHat 2.0.6 alignment, HTSeq gene counting and expression normalization using EdgeR $^{62}$. Gene expression is expressed in reads per kilobase pair per million mapped reads (RPKM)

Stable isotope labeling by amino acids in cell culture. Calvarial osteoblasts were isolated from Tgif1 ${ }^{-/}$mice and control littermates followed by expansion for 6 days in non-differentiation medium. Cells were re-plated at matching cell numbers for both genotypes not exceeding $80 \%$ confluence and cultured overnight. SILAC was performed for $12 \mathrm{~h}$ in DMEM (Gibco) supplemented with $0.1 \%$ dialyzed FBS lacking Met, Lys and Arg. Amino acid re-supplementation was achieved using L-Lys and L-Arg (Gibco) for control osteoblasts and 13C6 L-Lys and 13C6 L-Arg (Thermo Scientific) for Tgif1 ${ }^{-1-}$ osteoblasts. Met was replaced by L-Azidohomoalanin (AnaSpec) to allow for Click-iT-based enrichment. Conditioned medium obtained from Tgif1 $1^{-/}$and control osteoblasts was combined in a 1:1 ratio. Cell debris was removed by centrifugation and protease inhibitors (Roche) were added to the solution. Next, newly synthesized proteins were enriched from the conditioned medium using ultra centrifugal filters with a $3 \mathrm{kDa}$ cutoff (Amicon) and processed using the Click-iT Protein Enrichment-Kit (Thermo Fisher) according to the manufacturer's protocol.

Proteome analysis. For proteome analysis, proteins were subjected to in-solution digestion with trypsin. Briefly, proteins were reduced with $100 \mathrm{mM}$ dithiothreitol dissolved in $100 \mathrm{mM} \mathrm{NH}_{4} \mathrm{HCO}_{3}$ at $50^{\circ} \mathrm{C}$ for $10 \mathrm{~min}$, followed by alkylation with $300 \mathrm{mM}$ iodoacetamid dissolved in $100 \mathrm{mM} \mathrm{NH}_{4} \mathrm{HCO}_{3}$ for 30 min at room temperature. Proteins were incubated with trypsin for $16 \mathrm{~h}$ at $37^{\circ} \mathrm{C}$. The digests were acidified with formic acid (FA) and evaporated. For analysis of the tryptic peptides with liquid chromatography coupled to mass spectrometry (LC-MS), samples were dissolved in $20 \mu \mathrm{l} 0.1 \%$ FA. LC-MS measurements were performed by injecting the samples on a nano liquid chromatography system (Dionex UltiMate 3000 RSLCnano, Thermo Scientific) coupled via electrospray-ionization to a linear trap quadrupole orbitrap mass spectrometer (Orbitrap Fusion, Thermo Scientific) ${ }^{63}$. Peptides were separated by reversed phase chromatography (buffer A: $0.1 \% \mathrm{FA}$, buffer B: $99.9 \%$ CAN, $0.1 \% \mathrm{FA}$; flow-rate: $250 \mathrm{nl} / \mathrm{min}$; gradient: $2-30 \%$ B in 90 $\mathrm{min}$ ). Every second, a MS scan was performed over a $\mathrm{m} / \mathrm{z}$ range from 400 to 1500 , with a resolution of 120,000 FWHM (full width at half maximum) at $\mathrm{m} / z 200$ (transient length $=256 \mathrm{~ms}$, maximum injection time $=50 \mathrm{~ms}$, automatic gain control (AGC) target $=2 \mathrm{e} 5$ ). Analysis of fragmented peptide ions in the mass spectrometer was carried out in data-dependent acquisition mode, using the top speed mode, a HCD (higher-energy collisional dissociation) collision energy of $35 \%$, an intensity threshold of $5 \mathrm{e} 3$ and an isolation width of $1.5 \mathrm{~m} / \mathrm{z}$. Fragment spectra were recorded in the ion trap (scan-rate $=66 \mathrm{kDa} / \mathrm{s}$, maximum injection time $=70 \mathrm{~ms}$, AGC target $=1 \mathrm{e} 4$ ). LC-MS raw data were processed with Proteome Discoverer 1.4 (Thermo Scientific). For identification, fragment spectra were searched with Sequest HT against a mouse database (SwissProt, www.uniprot.org, downloaded 1.5.2014). The searches were performed using the following parameters: precursor mass tolerance was set to 10 parts per million (ppm) and fragment mass tolerance was set to $0.5 \mathrm{Da}$. Furthermore, two missed cleavages were allowed and a carbamidomethylation on cysteine residues as a fixed modification An oxidation of methionine residues and a ${ }^{13} \mathrm{C}_{6}$-label on both lysine and arginine residues were allowed as variable modifications. Peptides were identified with a false discovery rate of $1 \%$ using percolator and proteins were kept as correctly identified if at least two unique peptides were identified. For SILAC quantitation, event detector and precursor ion quantifier algorithms of Proteome Discoverer were used ${ }^{64}$. A mass variability of $2 \mathrm{ppm}$ and a $0.2 \mathrm{~min}$ retention time tolerance on precursor ion pairs were used and protein ratios were based on the median peptide ratio. At least two isotopic peaks were required for inclusion, as well as a minimal signal to noise level of three.

Immunoblotting. Cells were lysed in low salt mRIPA buffer ( $\mathrm{pH}$ 7.5) containing $50 \mathrm{mM}$ Tris base, $150 \mathrm{mM} \mathrm{NaCl}, 0.5 \%$ Nonidet P- $40,0.25 \%$ sodium deoxycholate and complete protease and phosphatase inhibitors (Roche). Lysates were separated on $12 \%$ polyacrylamide gels and subjected to immunoblot blot analysis. Immunoblots were incubated overnight at $4{ }^{\circ} \mathrm{C}$ with primary antibodies against Tgif1 (1:1000, rabbit monoclonal, Abcam, Cat. No: ab52955), Tgif2 (1:500, rabbit polyclonal, Millipore, Cat. No: 09-718), Sema3E (1:1000, goat polyclonal, R\&D Systems, Cat. No: AF3239), pCREB (1:500, rabbit monoclonal, Cell Signaling, Cat. No: 87G3), Mef2c (1:1000, rabbit monoclonal, Abcam, Cat. No: 197070) and Actin (1:5000, mouse monoclonal, Millipore, Cat. No: MAB1501). Peroxidase-labeled anti-rabbit or anti-mouse secondary antibodies (1:10,000, Promega, Cat. No: W401B, W402B) were used to visualize bands using the Clarity Western ECL Substrate (Bio-Rad). Immunoblot images were acquired using the ChemiDoc imaging system and Image Lab software (Bio-Rad). Unprocessed scans of the most important immunoblots are supplied in the source data file.

Immunohistochemistry. To determine sclerostin expression in mouse bones, mice were sacrificed $24 \mathrm{~h}$ after the last PTH injection and tibiae were fixed as described above. Bones were decalcified in 10\% EDTA ( $\mathrm{pH} \mathrm{7.4)}$ ) for 2 weeks, dehydrated, embedded in paraffin and cut in $4 \mu \mathrm{m}$ sections. Staining for sclerostin was performed using an anti-sclerostin antibody (1:50, goat polyclonal, R\&D Systems, Cat. No: AF1589).

ELISA. Enzyme-linked immunosorbent assay (ELISA) was used for quantitative determination of RANKL and OPG (both Immunodiagnostics systems) in mouse serum. All procedures were performed according to the manufacturer's guidelines.

Chromatin immunoprecipitation. Putative Tgif binding sites in the SOST promoter were identified using the online platform ALGGEN-PROMO. ChIP was performed using the A/G MAGNA ChIP kit (Millipore, Cat. No: 17-10085) according to the manufacturer's instruction. Briefly, $5 \times 10^{6}$ cells of the osteocytelike cell line OCY454 were plated into $15 \mathrm{~cm}^{2}$ dishes to differentiate for 2 weeks at $37^{\circ} \mathrm{C}$. After $2 \mathrm{~h}$ of treatment with either PTH $(100 \mathrm{nM})$ or vehicle, crosslinking was performed using $1 \%$ formaldehyde for $10 \mathrm{~min}$, followed by quenching for $5 \mathrm{~min}$ with glycin. After chromatin isolation, DNA was sheared into fragments ranging from $100 \mathrm{bp}$ to $500 \mathrm{bp}$ by 30 cycles of high-frequency sonication using a BioraptorPlus. ChIP was performed using $5 \mu \mathrm{g}$ of rabbit polyclonal anti-Tgif1 antibody (Santa Cruz Biotechnology, Cat. No: sc9084) or $5 \mu \mathrm{g}$ of a ChIP-grade polyclonal rabbit anti-IgG antibody (Abcam, Cat. No: ab37415). The amount of DNA pulled down was quantified by qPCR. All Ct values exceeding 34 were excluded from the analysis. ChIP-qPCR data were first normalized to the input of each precipitation using the formula (2(Ct 100\% input-Ct sample) $)$, followed by normalization to the IgG control. Rara was used as positive control ${ }^{55}$. The oligonucleotides used for amplifying the DNA fragments representing the 11 Tgifl binding sites are listed in Supplementary Table 10. A region nearby the transcriptional start site of the Sost gene was used as negative control.

Statistical analyses. Quantitative data are presented as mean \pm s.e.m. unless otherwise described. Parametric data were analyzed using an appropriate two-tailed Student's $t$-test when two groups were compared. A one-way analysis of variance (ANOVA) was used when more than two groups were compared, followed by Newman-Keuls post-hoc analysis to compare the groups. Probability values were considered statistically significant at $p<0.05$. Experiments were repeated at least three times as biological replicates with minimum of two technical replicates. No sample size calculations were performed but the number of mice analyzed in animal studies was determined in agreement with the standards in the field. Variation between groups was similar in all cases. Statistical analyses were performed using GraphPad Prism software.

Reporting summary. Further information on experimental design is available in the Nature Research Reporting Summary linked to this article.

\section{Data availability}

Next generation RNA-sequencing data that support the findings of this study have been deposited at the Center for Biotechnology Information with the accession code GSE89132. The mass spectrometry proteomics data that support the findings of this 
study have been deposited to the ProteomeXchange Consortium via the PRIDE partner repository with the dataset identifier PXD012303.

Received: 11 July 2017 Accepted: 29 January 2019

Published online: 22 March 2019

\section{References}

1. Edwards, M. H., Dennison, E. M., Aihie Sayer, A., Fielding, R. \& Cooper, C. Osteoporosis and sarcopenia in older age. Bone 80, 126-130 (2015).

2. Baron, R. \& Hesse, E. Update on bone anabolics in osteoporosis treatment: rationale, current status, and perspectives. J. Clin. Endocrinol. Metab. 97, 311-325 (2012).

3. Pisani, P. et al. Major osteoporotic fragility fractures: risk factor updates and societal impact. World J. Orthop. 7, 171 (2016).

4. Harada, S. \& Rodan, G. A. Control of osteoblast function and regulation of bone mass. Nature 423, 349-355 (2003).

5. Lian, J. B. et al. Networks and hubs for the transcriptional control of osteoblastogenesis. Rev. Endocr. Metab. Disord. 7, 1-16 (2006).

6. Marie, P. J. Transcription factors controlling osteoblastogenesis. Arch. Biochem. Biophys. 473, 98-105 (2008).

7. Baron, R. \& Kneissel, M. WNT signaling in bone homeostasis and disease: from human mutations to treatments. Nat. Med. 19, 179-192 (2013).

8. Hesse, E. et al. Zfp521 controls bone mass by HDAC3-dependent attenuation of Runx2 activity. J. Cell. Biol. 191, 1271-1283 (2010).

9. Quinn, J. M. W., Elliott, J., Gillespie, M. T. \& Martin, T. J. A combination of osteoclast differentiation factor and macrophage-colony stimulating factor is sufficient for both human and mouse osteoclast formation in vitro. Endocrinology 139, 4424-4427 (1998).

10. Takahashi, N., Udagawa, N. \& Suda, T. A new member of tumor necrosis factor ligand family, ODF/OPGL/TRANCE/RANKL, regulates osteoclast differentiation and function. Biochem. Biophys. Res. Commun. 256, 449-455 (1999).

11. Aubin, J. E. \& Bonnelye, E. Osteoprotegerin and its ligand: a new paradigm for regulation of osteoclastogenesis and bone resorption. Osteoporos. Int. 11, 905-913 (2000).

12. Sabokbar, A., Mahoney, D. J., Hemingway, F. \& Athanasou, N. A. Noncanonical (RANKL-Independent) pathways of osteoclast differentiation and their role in musculoskeletal diseases. Clin. Rev. Allergy Immunol. 51, 16-26 (2016).

13. Kawai, M. \& Rosen, C. J. The IGF-I regulatory system and its impact on skeletal and energy homeostasis. J. Cell. Biochem. 111, 14-19 (2010).

14. Solomon, C. G., Black, D. M. \& Rosen, C. J. Postmenopausal Osteoporosis. N. Engl. J. Med. 374, 254-262 (2016).

15. Eastell, R. \& Walsh, J. S. Anabolic treatment for osteoporosis: teriparatide. Clin. Cases Miner. Bone Metab. 14, 173 (2017).

16. Chen, P. et al. Increases in BMD correlate with improvements in bone microarchitecture with teriparatide treatment in postmenopausal women with osteoporosis. J. Bone Miner. Res. 22, 1173-1180 (2007).

17. Senn, C. et al. Comparative effects of teriparatide and ibandronate on spine bone mineral density (BMD) and microarchitecture (TBS) in postmenopausal women with osteoporosis: a 2-year open-label study. Osteoporos. Int. 25, 1945-1951 (2014).

18. Little, R. D. et al. A mutation in the LDL receptor-related protein 5 gene results in the autosomal dominant high-bone-mass trait. Am. J. Hum. Genet. 70, 11-19 (2002).

19. Boyden, L. M. et al. High bone density due to a mutation in LDLreceptor-related protein 5. N. Engl. J. Med. 346, 1513-1521 (2002).

20. Balemans, W. et al. The binding between sclerostin and LRP5 is altered by DKK1 and by high-bone mass LRP5 mutations. Calcif. Tissue Int. 82, 445-453 (2008).

21. $\mathrm{Li}, \mathrm{X}$. et al. Sclerostin binds to LRP5/6 and antagonizes canonical Wnt signaling. J. Biol. Chem. 280, 19883-19887 (2005).

22. Boudin, E. et al. The Lrp4 R1170Q homozygous knock-in mouse recapitulates the bone phenotype of sclerosteosis in humans. J. Bone Miner. Res. 32, 1739-1749 (2017).

23. Chang, M.-K. et al. Disruption of Lrp4 function by genetic deletion or pharmacological blockade increases bone mass and serum sclerostin levels. Proc. Natl. Acad. Sci. USA 111, E5187-E5195 (2014).

24. Balemans, W. et al. Increased bone density in sclerosteosis is due to the deficiency of a novel secreted protein (SOST). Hum. Mol. Genet. 10, 537-543 (2001).

25. Loots, G. G. et al. Genomic deletion of a long-range bone enhancer misregulates sclerostin in Van Buchem disease. Genome Res. 15, 928-935 (2005).
26. Ominsky, M. S. et al. Romosozumab improves bone mass and strength while maintaining bone quality in ovariectomized cynomolgus monkeys. J. Bone Miner. Res. 32, 788-801 (2017).

27. Cosman, F. et al. Romosozumab treatment in postmenopausal women with osteoporosis. N. Engl. J. Med. 375, 1532-1543 (2016).

28. Saag, K. G. et al. Romosozumab or alendronate for fracture prevention in women with osteoporosis. N. Engl. J. Med. 377, 1417-1427 (2017).

29. Kaplon, H. \& Reichert, J. M. Antibodies to watch in 2018. Mabs 10, 183-203 (2018).

30. Guo, J. et al. Suppression of Wnt signaling by Dkk1 attenuates PTH-mediated stromal cell response and new bone formation. Cell Metab. 11, 161-171 (2010).

31. Keller, H. \& Kneissel, M. SOST is a target gene for PTH in bone. Bone 37, 148-158 (2005).

32. Leupin, O. et al. Control of the SOST bone enhancer by PTH Using MEF2 transcription factors. J. Bone Miner. Res. 22, 1957-1967 (2007).

33. Hughes, A., Kleine-Albers, J., Helfrich, M. H., Ralston, S. H. \& Rogers, M. J. A class III semaphorin (Sema3e) inhibits mouse osteoblast migration and decreases osteoclast formation in vitro. Calcif. Tissue Int. 90, 151-162 (2012).

34. Shen, J. \& Walsh, C. A. Targeted disruption of Tgif, the mouse ortholog of a human holoprosencephaly gene, does not result in holoprosencephaly in mice. Mol. Cell. Biol. 25, 3639-3647 (2005).

35. Taniguchi, K. et al. Genetic and molecular analyses indicate independent effects of TGIFs on Nodal and Gli3 in neural tube patterning. Eur. J. Hum. Genet. 25, 208-215 (2017).

36. Rodda, S. J. \& McMahon, A. P. Distinct roles for Hedgehog and canonical Wnt signaling in specification, differentiation and maintenance of osteoblast progenitors. Development 133, 3231-3244 (2006).

37. Bivi, N. et al. Cell autonomous requirement of connexin 43 for osteocyte survival: Consequences for endocortical resorption and periosteal bone formation. J. Bone Miner. Res. 27, 374-389 (2012).

38. Sims, N. A. \& Martin, T. J. Coupling the activities of bone formation and resorption: a multitude of signals within the basic multicellular unit. Bone. Rep. 3, 481 (2014).

39. Sims, N. A. \& Walsh, N. C. Intercellular cross-talk among bone cells: new factors and pathways. Curr. Osteoporos. Rep. 10, 109-117 (2012).

40. Matsuo, K. \& Otaki, N. Bone cell interactions through Eph/ephrin. Cell Adh Migr. 6, 148-156 (2012).

41. Kudo, O. et al. Interleukin-6 and interleukin-11 support human osteoclast formation by a RANKL-independent mechanism. Bone 32, 1-7 (2003).

42. Zhang, M.-Z. et al. TGIF governs a feed-forward network that empowers Wnt signaling to drive mammary tumorigenesis. Cancer Cell 27, 547-560 (2015).

43. Wang, J.-L. et al. TGF $\beta$ induced factor homeobox 1 promotes colorectal cancer development through activating $\mathrm{Wnt} / \beta$-catenin signaling. Oncotarget 8, 70214-70225 (2017).

44. Cui, Y. et al. Lrp5 functions in bone to regulate bone mass. Nat. Med. 17, 684-691 (2011)

45. Lombardi, G. et al. The roles of parathyroid hormone in bone remodeling: prospects for novel therapeutics. J. Endocrinol. Invest. 34, 18-22 (2011).

46. Robling, A. G. et al. Anabolic and catabolic regimens of human parathyroid hormone 1-34 elicit bone- and envelope-specific attenuation of skeletal effects in Sost-deficient mice. Endocrinology 152, 2963-2975 (2011).

47. Stanislaus, D., Devanarayan, V. \& Hock, J. M. In vivo comparison of activated protein-1 gene activation in response to human parathyroid hormone (hPTH) (1-34) and hPTH(1-84) in the distal femur metaphyses of young mice. Bone 27, 819-826 (2000).

48. Saini, V. et al. Parathyroid hormone (PTH)/PTH-related peptide type 1 receptor (PPR) signaling in osteocytes regulates anabolic and catabolic skeletal responses to PTH. J. Biol. Chem. 288, 20122-20134 (2013).

49. O'Brien, C. A. et al. Control of bone mass and remodeling by PTH receptor signaling in osteocytes. PLoS ONE 3, e2942 (2008).

50. Suzuki, A. et al. PTH/cAMP/PKA signaling facilitates canonical Wnt signaling via inactivation of glycogen synthase kinase-3beta in osteoblastic Saos-2 cells J. Cell. Biochem. 104, 304-317 (2008).

51. Tobimatsu, T. et al. Parathyroid hormone increases beta-catenin levels through Smad3 in mouse osteoblastic cells. Endocrinology 147, 2583-2590 (2006).

52. Saidak, Z., Le Henaff, C., Azzi, S., Marty, C. \& Marie, P. J. Low-dose PTH increases osteoblast activity via decreased Mef2c/Sost in senescent osteopenic mice. J. Endocrinol. 223, 25-33 (2014).

53. Bellido, T. et al. Chronic elevation of parathyroid hormone in mice reduces expression of sclerostin by osteocytes: a novel mechanism for hormonal control of osteoblastogenesis. Endocrinology 146, 4577-4583 (2005).

54. Pessah, M. et al. c-Jun interacts with the corepressor TG-interacting factor (TGIF) to suppress Smad2 transcriptional activity. Proc. Natl Acad. Sci. USA 98, 6198-6203 (2001). 
55. Zhang, H. et al. Negative functional interaction of retinoic acid and TGF-beta signaling mediated by TG-interacting factor during chondrogenesis. Cell. Physiol. Biochem. 23, 157-164 (2009).

56. Wein, M. N. et al. SIKs control osteocyte responses to parathyroid hormone. Nat. Commun. 7, 13176 (2016)

57. Kramer, I., Loots, G. G., Studer, A., Keller, H. \& Kneissel, M. Parathyroid hormone (PTH)-induced bone gain is blunted in SOST overexpressing and deficient mice. J. Bone Miner. Res. 25, 178-189 (2010).

58. Delgado-Calle, J. et al. Control of bone anabolism in response to mechanical loading and PTH by distinct mechanisms downstream of the PTH receptor. J. Bone Miner. Res. 32, 522-535 (2017).

59. Dempster, D. W. et al. Standardized nomenclature, symbols, and units for bone histomorphometry: a 2012 update of the report of the ASBMR Histomorphometry Nomenclature Committee. J. Bone Miner. Res. 28, 2-17 (2013).

60. Bouxsein, M. L. et al. Guidelines for assessment of bone microstructure in rodents using micro-computed tomography. J. Bone Miner. Res. 25, 1468-1486 (2010).

61. Spatz, J. M. et al. The Wnt inhibitor sclerostin is up-regulated by mechanical unloading in osteocytes in vitro. J. Biol. Chem. 290, 16744-16758 (2015).

62. Dudakovic, A. et al. High-resolution molecular validation of self-renewal and spontaneous differentiation in clinical-grade adipose-tissue derived human mesenchymal stem cells. J. Cell. Biochem. 115, 1816-1828 (2014).

63. Kwiatkowski, M. et al. Homogenization of tissues via picosecond-infrared laser (PIRL) ablation: giving a closer view on the in-vivo composition of protein species as compared to mechanical homogenization. J. Proteom. 134, 193-202 (2016).

64. Scholten, A. et al. In-depth quantitative cardiac proteomics combining electron transfer dissociation and the metalloendopeptidase Lys-N with the SILAC mouse. Mol. Cell. Proteom. 10, O111.008474-O111.008474 (2011).

\section{Acknowledgements}

We are indebted to S. Schroeder, J. Pamperin and D. Brandt for technical assistance and to G. Arndt and P. Missberger for mouse husbandry. We are grateful to the University Medical Center Hamburg-Eppendorf Microscopy Imaging Facility (Umif) for technical support. We thank R. Baron and A. Atfi for support at early stages of the project and H. Kronenberg for helpful discussions. We are grateful to C. Walsh for providing Tgif1 $1^{+/-}$ and Tgif $1^{l l+}$ mice, S.J. Brandt for providing the Tgifl reporter constructs, L. Bonewald for providing the IDG-SW3 cell line and M. Kneissel as well as I. Kramer (Novartis) for providing the anti-sclerostin antibody and the hSOST-ECR5 reporter construct, and A. Hall and F. Bor (Mereo BioPharma) for allowing the continued use of the anti-sclerostin antibody. H.S. received a postdoctoral fellowship from the Japanese Society for the Promotion of Science. C.L.L. received a Marie Curie postdoctoral fellowship from the European Commission (658632). H. Schlüter acknowledges funding from the Helmholtz Gemeinschaft (VH-VI-523). A.J.v.W. obtained funding from the National Institutes of Health (NIH) through NIAMS (AR049069). T.A.G. received funding from the NIH through NCI (U01CA143057). H.T. was supported by postdoctoral fellowships from $\mathrm{EMBO}$ and the Humboldt Foundation and obtained funding from the Deutsche Forschungsgemeinschaft (TA 1154/1-1). E.H. acknowledges funding from the Deutsche Forschungsgemeinschaft (HE 5208/2-1, HE 5208/2-3 and HE 5208/3-1), the
Bundesministerium für Bildung und Forschung (01KU1401B), the Elsbeth Bonhoff Foundation (Project 83), the AO-Foundation (S-13-73H) and the European Commission (PCIG10-GA-2011-303722).

\section{Author contributions}

H.S. performed the in vivo animal experiments and bone histomorphometry, analyzed data and interpreted the results. A.G. designed and performed experiments, analyzed data and interpreted results. S.B. conducted the luciferase and ChIP assays, analyzed and interpreted these data. M.M. established and performed osteoclast assays. L.M. determined the induction of Tgifl expression by PTH and PKA. K.J. helped with the histology and performed the in vitro part of the SILAC assay. C.L.L. performed immunoblots of Sema3E expression. M.K. performed the SILAC analysis and interpreted the data. V.S. and P.D.P. quantified Tgifl expression in Dmp1-Cre $e^{T}$; $P T H R 1^{f l / f l}$ mice and analyzed the data. H. Schlüter designed and supervised the SILAC assay. T.B. provided Tgifl expression data in Dmp1-caPTHR1 mice. A.J.v.W. performed RNAseq analyses in BMSCs, analyzed the data and interpreted the results. K.S.M. and T.A.G. supported the initiation of the study and helped to design and interpret the experiments. H.T. designed and performed experiments, analyzed data, interpreted results and wrote the manuscript. E.H. conceived the project, designed experiments, analyzed data, interpreted results and wrote the manuscript.

\section{Additional information}

Supplementary Information accompanies this paper at https://doi.org/10.1038/s41467019-08778-x.

Competing interests: The authors declare no competing interests.

Reprints and permission information is available online at http://npg.nature.com/ reprintsandpermissions/

Journal peer review information: Nature Communications thanks the anonymous reviewers for their contribution to the peer review of this work

Publisher's note: Springer Nature remains neutral with regard to jurisdictional claims in published maps and institutional affiliations.

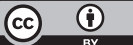

Open Access This article is licensed under a Creative Commons Attribution 4.0 International License, which permits use, sharing, adaptation, distribution and reproduction in any medium or format, as long as you give appropriate credit to the original author(s) and the source, provide a link to the Creative Commons license, and indicate if changes were made. The images or other third party material in this article are included in the article's Creative Commons license, unless indicated otherwise in a credit line to the material. If material is not included in the article's Creative Commons license and your intended use is not permitted by statutory regulation or exceeds the permitted use, you will need to obtain permission directly from the copyright holder. To view a copy of this license, visit http://creativecommons.org/ licenses/by/4.0/

(c) The Author(s) 2019 